\title{
KINEMATICS OF M51-TYPE INTERACTING GALAXIES
}

\author{
G. I. GÜNThardT ${ }^{1,5}$, R. J. Díaz ${ }^{2,3}$, AND M. P. AgüERo ${ }^{1,4}$ \\ ${ }_{1}$ Observatorio Astronómico, Universidad Nacional de Córdoba, Laprida 854, 5000 Córdoba, Argentina; \\ guillermo.gunthardt@unc.edu.ar, mpaguero@.oac.uncor.edu \\ ${ }^{2}$ Gemini Observatory, AURA, USA; rdiaz@gemini.edu \\ ${ }^{3}$ ICATE, CONICET, Argentina \\ ${ }^{4}$ CONICET, Argentina \\ Received 2016 March 20; revised 2016 July 19; accepted 2016 July 20; published 2016 October 31
}

\begin{abstract}
We present a kinematic catalog for 21 M51-type galaxies. It consists of radial velocity distributions observed with long-slit spectroscopy along different position angles, for both the main and satellite components. We detect deviations from circular motion in most of the main galaxies of each pair, due to the gravitational perturbation produced by the satellite galaxy. However, some systems do not show significant distortions in their radial velocity curves. We found some differences between the directions of the photometric and kinematic major axes in the main galaxies with a bar subsystem. The Tully-Fisher relation in the $B$-band and $K s$-band for the present sample of M51type systems is flatter than in isolated galaxies. Using the radial velocity data set, we built a synthetic normalized radial velocity distribution, as a reference for future modeling of these peculiar systems. The synthetic rotation curve, representing the typical rotation curve of the main galaxy in an M51-type pair, is near to solid body-like inside $4 \mathrm{kpc}$, and then is nearly flat within the radial range $5-15 \mathrm{kpc}$. The relative position angles between the major axis of the main galaxy and the companion's location, as well as the amplitude of the velocity difference, indicate that the orbital motion of the satellite has a large projection on the equatorial plane of the main galaxy. In addition, the differences in radial velocity between the two galaxies indicate that the satellite's orbital motion is within the range of amplitudes of the rotation curve of the main galaxy, and all the M51-type systems studied here, except for one, are gravitationally bound.
\end{abstract}

Key words: galaxies: interactions - galaxies: kinematics and dynamics

\section{INTRODUCTION}

M51-type systems are interacting galaxies composed of a larger main galaxy and a smaller or satellite galaxy near to or at the end of a tidal arm developed by the larger component. Vorontsov-Velyaminov (1975) considered a subset of 160 interacting galaxies of this type, and many of these objects have been included in his "Atlas of Interacting Galaxies" (1977). Halton Arp also included 54 of these systems in the "Atlas of Peculiar Galaxies" (Arp 1966) and they are considered as a category of interaction in the "Catalog of Southern Peculiar Galaxies and Associations" of Arp \& Madore (1987). Jokimäki et al. (2008) built a catalog of more than 200 apparently interacting galaxy pairs of the M51 class, from visual identification of IRSA archives, and found that a low number of the main galaxies in M51 systems are early-type spirals and barred spirals and that about 70\% of the main galaxies in M51 systems are two-armed spirals.

M51-type galaxies are a subset of the class of unequal-mass interacting galaxy pairs, for which there is some statistical evidence based on large surveys for enhanced star formation relative to normal galaxies, particularly in the smaller of the galaxies in the pair (e.g., Woods \& Geller 2007; Ellison et al. 2008).

Ellison et al. (2008), from a sample of 1716 galaxies that are members of galaxy pairs from the Sloan Digital Sky Survey Data Release 4, found that the enhancement in star-forming rate

\footnotetext{
${ }^{5}$ Visiting astronomer at the Complejo Astronómico El Leoncito (CASLEO) operated under agreement between the Consejo Nacional de Investigaciones Científicas y Técnicas de la República Argentina and the National Universities of La Plata, Córdoba and San Juan.
}

(SFR) can be detected for a sample of galaxy pairs whose masses are within a factor of 10 of each other.

According to Smith et al. (2007), an M51-like subset of Arp galaxies also shows possible differences from the spirals in mid-infrared colors; however, the M51 sample size is small, so these results are tentative. For eight M51-like systems they obtain redder [8]-[24], [3.6]-[24], and [5.8]-[8] colors, when compared to spirals, and therefore enhanced star formation. The estimated median SFR (SFR derived from the $24 \mu \mathrm{m}$ luminosity, by using the calibration from Calzetti et al. 2005) for their small sample of M51-type galaxies was 3.3 $M_{\odot} \mathrm{yr}^{-1}$, while the median SFR of the spirals of their control sample is $0.9 M_{\odot} \mathrm{yr}^{-1}$. For comparison, these authors also find an SFR of $\sim 1.7 M_{\odot} \mathrm{yr}^{-1}$ for their sample of 35 tidally distorted premerger interacting galaxy pairs, which were selected from the Arp Atlas. For the mentioned sample of 35 Arp systems, they derived an SFR of $\sim 2.6 M_{\odot} \mathrm{yr}^{-1}$ from the $8-1000 \mu \mathrm{m}$ total infrared luminosity as a star-forming indicator.

Laurikainen et al. (1998) obtained deep broad-band BVRI photometry of close interacting galaxy pairs and found that nine of 13 M51-type pairs showed an enhanced star formation in the central regions of the companions, and only one pair in the nucleus of the main galaxy.

Klimanov \& Reshetnikov (2001), made a final selection of 32 M51-like objects from the list of Vorontsov-Velyaminov and performed a statistical study using photometric information from the B-band of the "Digitized Sky Survey" (DSS) and farinfrared data from the IRAS satellite. These authors conclude that there is an enhancement of the star-forming activity with respect to isolated galaxies. In fact, from IRAS data, they obtained an average SFR for the main galaxies of M51-type systems of $9 M_{\odot} \mathrm{yr}^{-1}$. They compare these results with those of 
Table 1

List of Objects

\begin{tabular}{|c|c|c|c|c|c|c|}
\hline Object & R.A. (J2000) & Decl. (J2000) & $\overline{B_{\text {main }}}$ & $\overline{B_{\text {sat }}}$ & $\overline{K s_{\text {main }}}$ & $\overline{K s_{\text {sat }}}$ \\
\hline NGC 633 & 013623.4 & -371918 & 13.33 & 15.15 & 9.92 & 10.84 \\
\hline ARP 54 & 022402.6 & -044136 & 14.87 & 16.33 & 10.91 & 13.04 \\
\hline AM $0327-285$ & 032956.1 & -284614 & 14.06 & 14.55 & 11.55 & 12.78 \\
\hline AM 0403-604 & 040427.1 & -604057 & 15.60 & 16.81 & 11.46 & 13.01 \\
\hline AM 0430-285 & 043211.3 & -285139 & 15.84 & $\ldots$ & 11.52 & 12.99 \\
\hline AM 0458-250 & 050041.9 & -250433 & 14.95 & 18.32 & 10.85 & 13.88 \\
\hline AM 0459-340 & 050141.2 & -340156 & 14.27 & $\ldots$ & 11.42 & 14.60 \\
\hline ESO 362-IG001 & 050155.9 & -340143 & 15.08 & $\ldots$ & 11.01 & 16.49 \\
\hline AM 0639-582 & 064043.2 & -583128 & 13.40 & 15.08 & 9.07 & 11.80 \\
\hline VV 410 & 104407.0 & -162811 & 14.50 & 16.01 & 11.07 & 12.50 \\
\hline VV 350 & 114011.4 & +152005 & 13.93 & 13.95 & 9.15 & 10.99 \\
\hline NGC 4188 & 121407.3 & -123510 & 14.57 & $\ldots$ & 10.89 & 16.68 \\
\hline AM 1304-333 & 130708.8 & -335158 & 14.71 & 16.30 & 10.92 & 11.48 \\
\hline AM $1325-274$ & 132803.3 & -275500 & 15.10 & $\ldots$ & 11.48 & 14.81 \\
\hline AM 1416-262 & 141922.4 & -263841 & 13.83 & $\ldots$ & 9.57 & 11.95 \\
\hline AM $1427-432$ & 143018.0 & -433340 & 14.96 & $\ldots$ & 10.96 & $\ldots$ \\
\hline VV 452 & 160116.3 & +174040 & 15.29 & $\ldots$ & 12.22 & $\ldots$ \\
\hline AM $1955-170$ & 195929.7 & -565957 & $\ldots$ & $\ldots$ & 12.13 & 12.77 \\
\hline AM 2058-381 & 210139.1 & -380459 & 15.43 & & 11.88 & 13.55 \\
\hline AM $2105-332$ & 210805.5 & -331355 & 13.90 & 15.15 & 9.17 & 11.38 \\
\hline AM 2256-304 & 225858.6 & -302938 & 14.53 & 18.25 & 11.07 & 14.97 \\
\hline
\end{tabular}

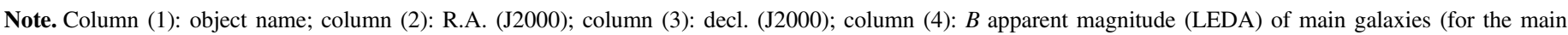

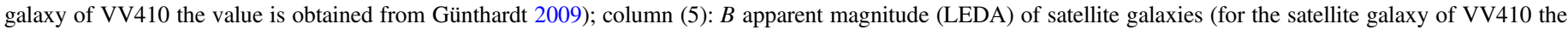

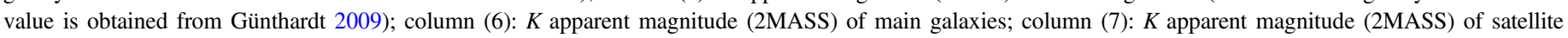
galaxies.

Bushouse (1987) for isolated galaxies, finding that the average SFR for this sample of M51-type galaxies is about seven times that of isolated galaxies. In summary, broad-band photometry surveys indicate that M51 galaxies are places of active star formation.

From the kinematic perspective, which is our main goal in the present work, we can assert that M51-like galaxies are not well studied systems. The kinematic data for objects of this type are scarce, and generally, for many of them, only the systemic velocity of the large galaxy is available. A long-slit spectroscopic kinematic study was previously performed by Klimanov et al. (2002), who obtained kinematic data for 13 M51-type systems and considered radial velocity determinations from the literature for eight other systems. From the mentioned data, Reshetnikov \& Klimanov (2003) found that moderately massive dark halos surround bright spiral galaxies and that the Tully-Fisher relation for M51-like galaxies was flatter than in local field galaxies. Other kinematic studies of this class of objects have been carried out from observations using Fabry-Perot interferometry. Rampazzo et al. (2005) have used the $\mathrm{H} \alpha$ emission line to map the warm gas distribution and the velocity fields of two M51-like systems, Arp 70 and Arp 74. They detected gas motions following the elongated arm/tail of Arp 70b, while in the fainter member, Arp 70a, the gas distribution is off-center with respect to the stellar isophotes, suggesting to the authors that it could be due to an external acquisition. They also detected non-circular motions in the velocity field of the main galaxy of Arp 74, Arp 74a. Fuentes-Carrera et al. (2007) studied the kinematics and dynamics of the M51-type pair NGC 3893/96, also using Fabry-Perot interferometry, and detected non-circular motions in the velocity fields of both galaxies, probably due to the encounter.
In this paper we aim to determine how common the kinematic perturbations reported before are, as well as to characterize the typical rotation curve and range of masses, using detailed observations of a relatively large sample of M51 candidate objects.

\section{OBSERVATIONS}

In what follows, we present a kinematic catalog for 21 M51type interacting systems (Table 1) corresponding to spectroscopic observations performed at "Complejo Astronómico El Leoncito," San Juan, Argentina, in six observing runs from 2005 to 2007 (April and October). We used the $2.15 \mathrm{~m}$ telescope and a REOSC spectrograph, in long-slit mode. A 1200 line $\mathrm{mm}^{-1}$ grating was used, and the resulting spectral resolution was about $3 \AA$. The seeing during observations fluctuated between $2^{\prime \prime}$ and $3^{\prime \prime}$, so the slit width was opened to $250 \mu \mathrm{m}$ or $300 \mu \mathrm{m}$, according to the observing conditions. The wavelength range was between $\sim 6200$ and $7000 \AA$. An example spectrum is shown in Figure 3. Generally, the slit was positioned on the nuclear region of both components of the M51-type systems and along the photometric main axis of both galaxies. Additional directions were chosen for some objects. Data reduction of the spectra employed the standard methods of the Image Reduction and Analysis Facility (IRAF) ${ }^{6}$ reduction package. Radial velocities were obtained by fitting a Gaussian profile to the $\mathrm{H} \alpha$ emission line, while uncertainties were determined from the empirical expression for $\sigma$ by Keel (2004). An uncertainty of $2 \sigma$ was assigned for each measured radial velocity.

\footnotetext{
6 http://iraf.noao.edu/ IRAF is distributed by the National Optical Astronomy Observatory, which is operated by the Association of Universities for Research in Astronomy (AURA) under a cooperative agreement with the National Science Foundation.
} 


\section{RESULTS}

In Table 1 we listed the selected objects, with their absolute equatorial coordinates and $B$-band magnitudes, which have been extracted from LEDA (Lyon-Meudon Extragalactic Database). ${ }^{7}$ The $B$-band images of these objects are shown in Figure 1. We have measured $K s$-band total integrated magnitudes (aperture method) for both main and satellite galaxies from the corresponding calibrated 2MASS images, since generally there is no information for satellite galaxies in the 2MASS photometric catalogs. Details of the photometric procedure may be found in Günthardt (2009). In Table 1 we include $K s$-band apparent magnitudes for main and satellite galaxies of the sample and in Figure 2 we show histograms corresponding to the $K s$-band (2MASS) luminosity for main galaxies and $K s$ luminosity ratios between satellite and main galaxies of this sample. Ks-band magnitudes have been corrected for Galactic extinction (Schlafly \& Finkbeiner 2011), while internal extinction has been estimated by following the expressions of Masters et al. (2003, see their Table 5). For the $k$-correction $\left(k_{K}\right)$ we considered a linear fit for the low-redshift end of the models of Poggianti (1997), where $k_{K}=-1,52 z$. The cosmological constant has been adopted as $H_{0}=75 \mathrm{~km} \mathrm{~s}^{-1} \mathrm{Mpc}^{-1}$ and the $K$-band luminosities have been determined using $L_{K}=10^{-0.4\left(M_{K}-3.28\right)}$.

In Figure 1, we plot the $B$-band DSS images and the positions of the slit. Figure 4 shows, for the different observed position angles, the heliocentric radial velocity distributions (not corrected for the inclination of the disks with respect to the line of sight). In some cases, the observed radial velocity gradients are drawn. In Table 2 we present the systemic velocities corresponding to main and satellite components, and in Figure 2 we show a histogram of the systemic differences in radial velocity between main and satellite galaxies. For each observed system presented in this catalog, a qualitative kinematic analysis was done. We also present a comparison between kinematic and photometric major axes. The TullyFisher relation (Figure 7) is plotted with radial velocity data derived from this work and $B$-band and $K s$-band magnitudes. Finally synthetic radial velocity curves are derived for the main galaxies.

\subsection{General Morphological Characteristics of the Sample}

We present a brief description of the morphology of the systems and search for common characteristics by considering the projected separation between components, the development of the tidal arms, and the inclination of the disks of the components.

Some of the studied interacting systems have a very well developed tidal arm while their satellites have a disk-like structure and are nearly edge-on. Examples of this group are VV 410, NGC 633, AM 0459-340, and AM 2058-381. Other systems present a well developed tidal arm, with satellites having E-type or irregular morphologies, such as AM 0639-582 and AM 0430-285. The opposite case is represented by some pairs that are apparently very near to each other, such as NGC 4188, VV 452, AM 1325-274, and AM 0403-604. It is also possible to find similar configurations in AM 1304-333 and AM 0327-285, but now with satellites having a considerable mass in relation to the mass of their main galaxies. There

\footnotetext{
7 http://leda.univ-lyon1.fr/
}

are intermediate cases in which a poorly developed tidal arm is seen, with an apparent lack of contact with the satellite: AM 1416-262 and ARP 54. The main galaxy in Arp 54 is a typical ocular-shaped galaxy, that is, a galaxy that contains a central transient eye-shaped structure. This may be the result of an encounter with a smaller galaxy (for more details, see Thomasson 2004). Other subgroups include systems where their tidal arms are rather diffuse, such as AM 2103-332 and VV 350. With regard to nuclear activity, the main galaxy of AM 0403-604 is the only Seyfert 2 type galaxy in this kinematic sample (Günthardt 2009); it has an edge-on close companion and, in addition to the tidal arm, a bridge is seen connecting the galaxies. AM 1416-262 has a main galaxy that is the only Seyfert 1 in the sample.

\subsection{Analysis by Object}

$N G C 633$. In the DSS picture, the main galaxy presents two well developed arms. Both arms end in a diffuse fashion, near the satellite galaxy, so it would be possible to consider this object as an atypical M51-type system, taking into account that it is the shorter arm that is closer in projection to the satellite galaxy. Apparently, the satellite would be the cause of the longer extension that presents the longer tidal arm. This tidal arm seems to begin in the southwestern part of the disk of the main galaxy, although it is difficult to locate exactly in which part of the disk it originates, because of its very diffuse structure. The longer arm could have been disturbed by the passage of the satellite galaxy, and actually, the smaller galaxy would almost coincide with the position of the shorter, and less perturbed arm, which originates in the eastern side of the disk of the main galaxy. From $\mathrm{H} \alpha$ images obtained by Dopita et al. (2002), we observed that the emission is concentrated in the nuclear regions of both galaxies and that the distribution of the $\mathrm{H} \alpha$ emission in the satellite is perpendicular to its major axis, as was noticed by Dopita et al. (2002). As these authors pointed out, this could be due to the presence of outflow. In what follows, we analyze the radial velocity data for different position angles (PA).

PA $136^{\circ}$ : The observed gradient in the central kiloparsec $\left(7^{\prime \prime}\right)$ is $55 \mathrm{~km} \mathrm{~s}^{-1} \mathrm{kpc}^{-1}$. If we take into account the whole rigidbody extent of the curve, the gradient is about $30 \mathrm{~km} \mathrm{~s}^{-1} \mathrm{kpc}^{-1}$. The general aspect of the curve is rather normal.

PA $90^{\circ}$ : We noted some disturbances in this position angle. From $-5 \mathrm{kpc}\left(-14^{\prime \prime}\right)$ until a position near the center, we measure a gradient of $10 \mathrm{~km} \mathrm{~s}^{-1} \mathrm{kpc}^{-1}$, while from the center to $3 \mathrm{kpc}\left(9^{\prime \prime}\right)$ it is about $30 \mathrm{~km} \mathrm{~s}^{-1} \mathrm{kpc}^{-1}$. Beyond the central $10^{\prime \prime}$ the radial velocity gradients decrease and the variations reach $75 \mathrm{~km} \mathrm{~s}^{-1}$, which may be associated with the arms, although these significant amplitude variations may be due to the gravitational perturbation of the satellite. We also found asymmetries in the radial velocity distributions along $\mathrm{PA}=53^{\circ}$.

For this system, we have obtained spectra for three directions, so it was possible to determine its main kinematic axis, which is coincident with its main photometric axis. Generally, some mild asymmetries and distortions are observed in the radial velocity distribution of the main galaxy.

The radial velocity distribution along the main axis of the satellite is rigid body-like over practically its whole extent.

$A R P$ 54. The main galaxy is classified as $\mathrm{SABc}$ and presents an "ocular" morphology, a denomination that makes reference to the apparent shape of the ring of the galaxy and the nuclear region. The difference in radial velocity between the two components is $\sim 60 \mathrm{~km} \mathrm{~s}^{-1}$ (the radial velocity of the satellite 

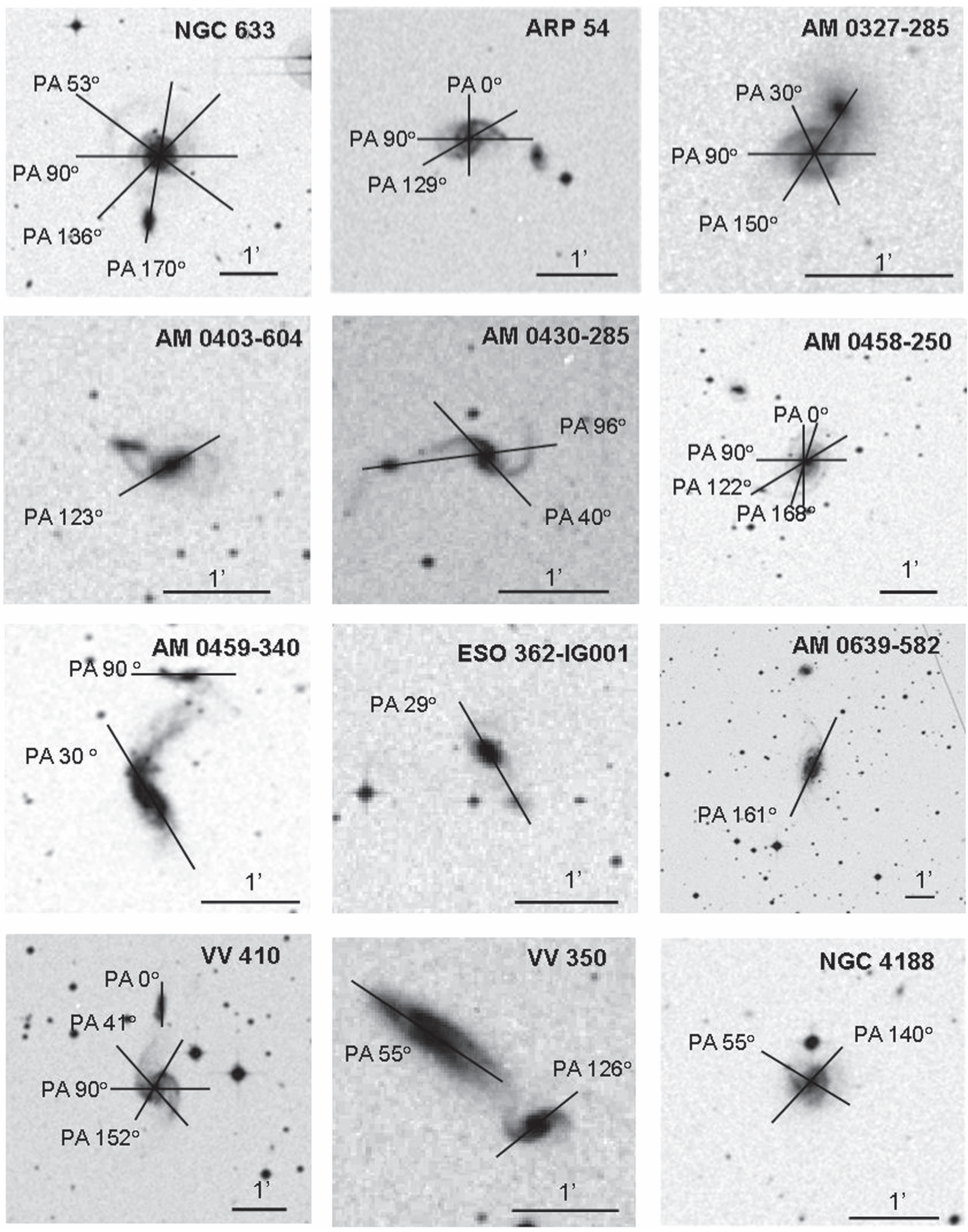

Figure 1. B-band images from the "Digitized Sky Survey." The dark segments mark the position angles of the obtained spectra. 

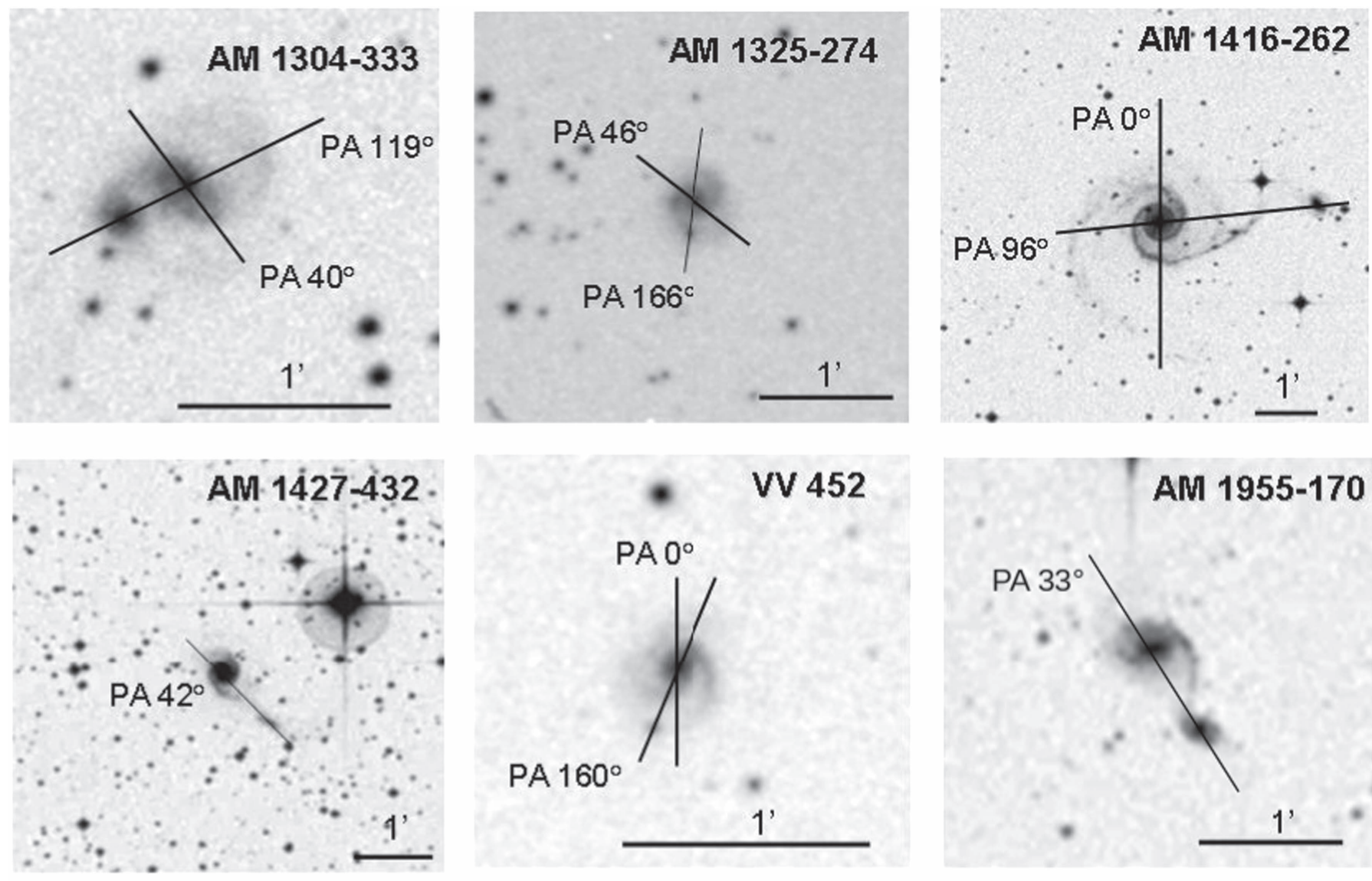

AM 1955-170
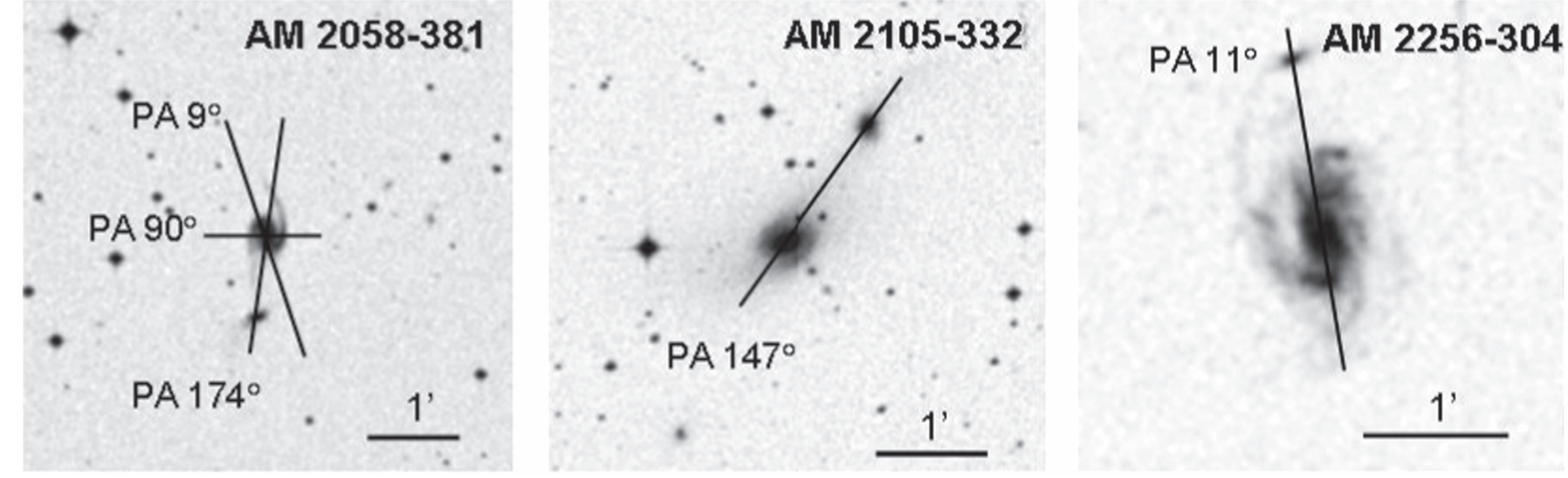

Figure 1. (Continued.)

had no previous determinations and has been determined in CASLEO with the same instrumental configuration described above, but with a 300 line $\mathrm{mm}^{-1}$ grating). The arm that would connect with the satellite is abruptly interrupted, in both 2MASS and DSS images. The radial velocity distribution along PA $90^{\circ}$ for the main galaxy was measured from very faint $\mathrm{H} \alpha$ emission in its central $6^{\prime \prime}$, so it was not possible to derive kinematic information for the central region. The curve does not present significant distortions: the variations observed beyond $10^{\prime \prime}$ in the western direction correspond to the arm positions (and to the position of an emission region placed between the disk and the western arm). Only a slight asymmetry is noted in PA $0^{\circ}$ : the southern region of the curve is almost constant and it rises by $50 \mathrm{~km} \mathrm{~s}^{-1}$ in the northern part, precisely from the position where the tidal arm begins.
This asymmetry is the only sign of perturbation due to the satellite.

AM 0327-285. This system presents a relatively small separation between the components. In the direction that connects the two objects, the distribution has an observed maximum of $250 \mathrm{~km} \mathrm{~s}^{-1}$ with some slight asymmetries, such as a rise in the radial velocity toward the direction of the satellite. The perturbation perhaps is more marked along PA $30^{\circ}$, where the peak of the continuum emission coincides with the position of the maximum in radial velocity. From this position, to the $\mathrm{SW}$, the curve decreases by about $100 \mathrm{~km} \mathrm{~s}^{-1}$. For PA $90^{\circ}$, we have found variations of about $100 \mathrm{~km} \mathrm{~s}^{-1}$, which correspond spatially to the position of the spiral arms.

AM 0403-604. In this system, besides its M51-type nature, a bridge apparently connects the two galaxies. The main galaxy 

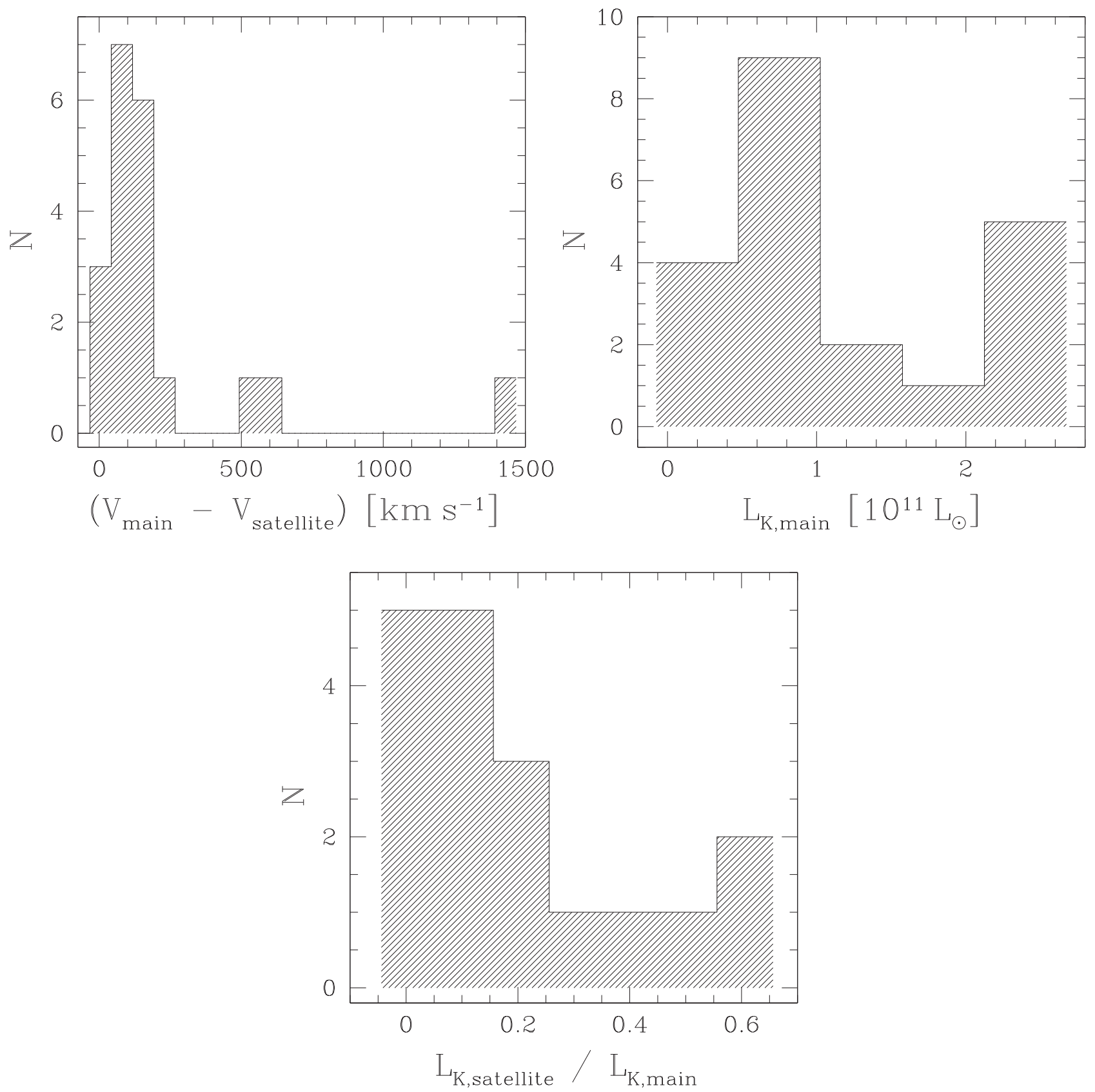

Figure 2. Upper left: distribution of the modulus of the difference in radial velocity between main and satellite galaxies. Upper right: distribution of the $K s$-band luminosity (2MASS) for the main galaxies of the present sample. Bottom: distribution of the ratio of luminosities in the Ks-band (2MASS) for the Ks-band luminosity of the satellite galaxy with respect to that of the main galaxy.

has a nuclear emission corresponding to Seyfert 2 type (as determined from spectrophotometric observations performed at CASLEO in 2007 October). The velocity difference between main and satellite galaxies, which had no previous determinations, is rather high, being $490 \mathrm{~km} \mathrm{~s}^{-1}$. It suggests that one of two active objects in the sample presents a direct connection (bridge) between the central region of the galaxy and its companion.

AM 0430-285. The morphology of this system is similar to that of NGC 646, an M51 system that is not included in this work but observed in CASLEO for a spectrophotometric study. A small condensation joined by a faint bridge/arm to the satellite galaxy is observed. This object did not have previous radial velocity measurements; the present work confirms that it constitutes a physical pair with the main galaxy. The direction that connects the center of the main and satellite galaxies coincides with the major axis of the satellite, which presents rotation, with an amplitude of $60 \mathrm{~km} \mathrm{~s}^{-1}$. The orbit of the system is prograde, and the radial velocity distribution of the main galaxy is asymmetric with respect to the position of the continuum emission peak. For the direction along the bar, which is nearly coincident with its photometric major axis, the velocity distribution presents pronounced breaks. The variations outside the rigid-body zone reach $70 \mathrm{~km} \mathrm{~s}^{-1} \mathrm{NE}$ from the center. If we take into account that the semi-amplitude of the rotation curve is $140 \mathrm{~km} \mathrm{~s}^{-1}$, the results are very significant.

AM 0458-250. The main galaxy of this system is large (according to LEDA data, its $25 \mathrm{mag} \operatorname{arcsec}^{-2}$ diameter in the 


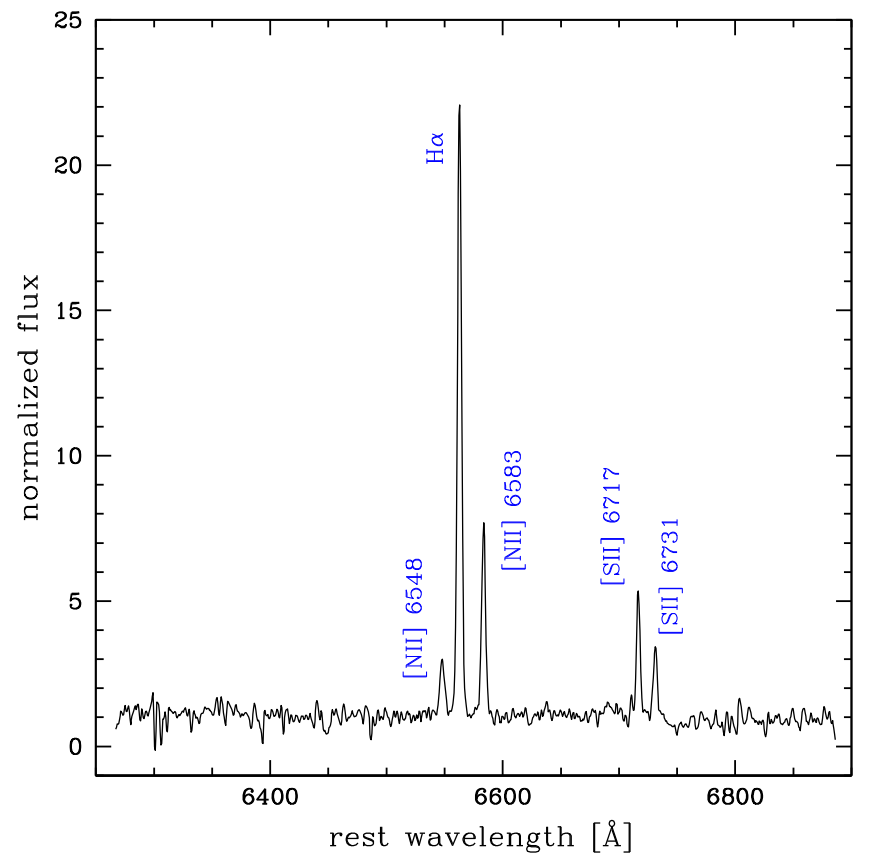

Figure 3. Example of an optical spectrum obtained with the REOSC spectrograph, in CASLEO, Argentina, with a 1200 line $\mathrm{mm}^{-1}$ grating. It corresponds to the nuclear region of the main galaxy of AM 0450-340. The spectrum extraction is 3 arcsec wide. The ordinate is in arbitrary units and is normalized to the spectral continuum.

$B$-band is $1 ! 15$ or about $50 \mathrm{kpc}$ ). The main galaxy is a massive spiral (the mass may be estimated from the velocity distribution, in a Keplerian approximation). Its satellite had no previous velocity determination and they are confirmed as a physical pair though no significant distortions are seen in the velocity distribution.

AM 0459-340. The heliocentric radial velocity curve, along the major axis of the main galaxy, presents high asymmetry with respect to the position of the nuclear region. The observed radial velocity gradient of the NE branch is $35 \mathrm{~km} \mathrm{~s}^{-1} \mathrm{kpc}^{-1}$, reaching a maximum $10^{\prime \prime}$ from the center, and with a difference of $80 \mathrm{~km} \mathrm{~s}^{-1}$ with respect to that of the central part. Here we find that the satellite forms a physical pair with the main galaxy. The satellite displays a rigid-body curve over most of its extent. The radial velocity gradient increases notably in the region of the bridge that connects with the eastern condensations, so it is possible that this small object was not previously linked with the satellite galaxy.

ESO 362-IG01. This system, 3' away from AM 0459-340, was not included previously in the M51-type category of interacting systems. No significant distortions are observed although an asymmetry in the $\mathrm{H} \alpha$ emission can be observed. This emission is more intense on the opposite side to the satellite and is the only part of the curve with a flat distribution (the outermost regions of the NE branch of the curve). For the other parts, the curve presents a rigid-body distribution. The satellite had no previous radial velocity measurements, so we confirm the physical link between the two components.

AM 0639-582. This system has a long tidal tail, which extends from the main spiral galaxy to an irregular galaxy. The radial velocity distribution along the major axis of the main galaxy is apparently normal, but there is a well marked difference in the observed gradients, the gradient being more pronounced in the satellite direction, between $4^{\prime \prime}$ north from the center and $3^{\prime \prime}$ to the south, and lower between $4^{\prime \prime}$ and $18^{\prime \prime}$ to the south. Observations along the direction that connects the centers of the two galaxies were also made, determining that the orbit is prograde. The radial velocity of the satellite is similar to the velocity that corresponds to the NW branch of the radial velocity distribution of the main galaxy. The observed radial velocity gradient of the central kiloparsec region is quite high, $135 \mathrm{~km} \mathrm{~s}^{-1} \mathrm{kpc}^{-1}$.

$V V 410$. The radial velocity distributions have been obtained for both objects along their main axes. The satellite galaxy has a rigid-body velocity distribution along almost the whole main body. Considering that the smaller galaxy is nearly edge-on, it is not possible to determine whether the shape of the radial velocity curve obeys a matter distribution dominated by a spheroidal component or whether it is a consequence of dust opacity (see Díaz et al. 2001 for a similar case). The main galaxy shows a velocity distribution along the bar orientation with a velocity amplitude and aspect that are typical of spiral galaxies of this morphological type. The most perturbed side of the curve is toward the satellite galaxy.

$V V$ 350. This system consists of a main galaxy of $\mathrm{SABb}$ type, nearly edge-on, and a satellite $\mathrm{SBb}$, also edge-on, and no kinematic information has been available until now. We obtained spectra for the major axis of both galaxies. It is interesting to notice how the disk of the main galaxy (seen in optical images) is better defined in the zone opposite to the satellite, while the region nearer to the companion appears diffuse or perturbed, perhaps by the effect of the interaction. The $\mathrm{H} \alpha$ emission is higher in the direction opposite to the satellite than toward it. The velocity curve of the main galaxy has rigid-body rotation over most of the extent of the main body, although it reaches a peak toward the NE, opposite to the satellite direction, while the curve in the satellite direction remains solid-body. The satellite velocity distribution has asymmetries, mainly toward the southeast, which is the region where the arm of the satellite begins that is in contact with the arm of the main galaxy.

$N G C 4188$. The satellite galaxy is located at the end of an almost undistorted arm. With no previous radial velocity determination for the small object, its physical link with the major galaxy is confirmed in this work. As in the case of VV 452, the radial velocity of the satellite is a continuation of the velocity distribution of the main galaxy. For the position angle that connects the two objects we observed some asymmetries. The radial velocity values reach a maximum at $4^{\prime \prime}$ northeast (that is, in the direction of the satellite) and the radial velocity distribution decreases thereafter.

$A M$ 1304-333. It is clearly seen in the DSS images that the nucleus of the main galaxy is apparently displaced with respect to the geometrical center of the disk of the main galaxy. In 2010 we obtained broad-band images with the Swope $1 \mathrm{~m}$ telescope of Las Campanas Observatory. These showed that the central region consists of a nest of bright knots. This system seems to be an extreme case in mass ratio, morphology, and kinematic perturbations. The blue DSS images depict a very perturbed disk with plumes. PA $40^{\circ}$ : the amplitude of the radial velocity curve is $172 \mathrm{~km} \mathrm{~s}^{-1}$. The velocity gradient of $120 \mathrm{~km} \mathrm{~s}^{-1} \mathrm{kpc}^{-1}$ is quite pronounced. The continuum emission peak is coincident with the "turnover" and the offcentering is $2^{\prime \prime}$. In the northeast direction the curve remains flat, except at $10^{\prime \prime}$ from the center, where a depression of about $60 \mathrm{~km} \mathrm{~s}^{-1}$ is observed. This depression could be associated 

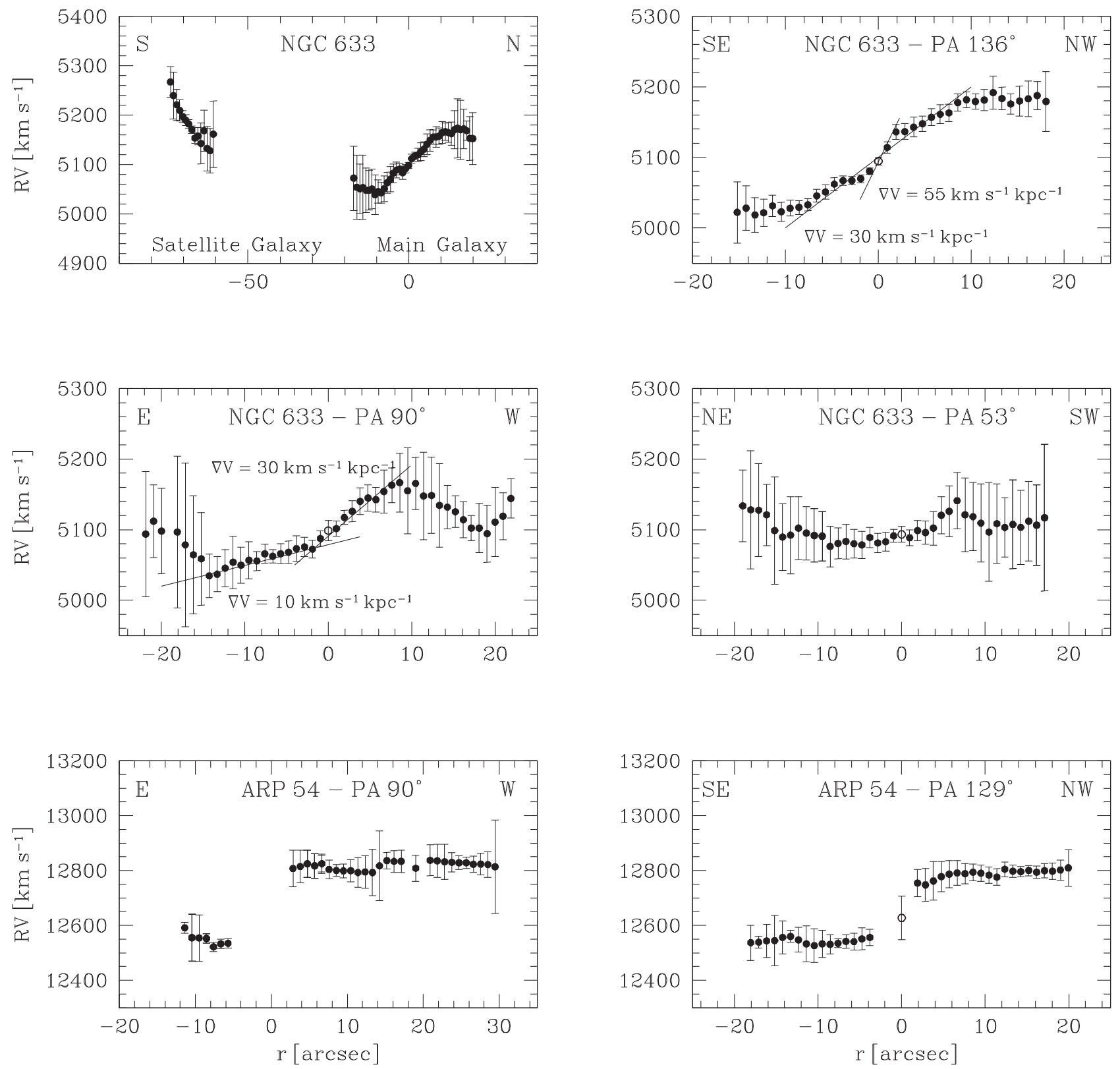

Figure 4. Heliocentric radial velocity distributions for objects in the catalog of M51-type galaxies. Open circles mark the location of the continuum emission peak in the spectra in the rest-frame wavelength range $6400-6800 \AA$. In some cases, the best fit to the velocity gradient is drawn as a straight line.

with the start of the spiral arm at the tip of the bar. To the SW and after the turnover, the radial velocity curve decreases between $8^{\prime \prime}$ and $15^{\prime \prime}$ (dust presence associated with arms?).

PA along both components: the main galaxy has a rotation curve that is notably distorted. The velocity amplitude of the main galaxy is $75 \mathrm{~km} \mathrm{~s}^{-1}$, without correction for inclination.

$A M$ 1325-274. The main galaxy seems to be quite face on. The velocity distribution at PA $166^{\circ}$ has a steady decrease with oscillations of $40 \mathrm{~km} \mathrm{~s}^{-1}$. The velocity of the satellite (which had no previous determinations) seems to be a continuation of the rotation curve of the main galaxy.

AM 1416-262. The main galaxy of this system is classified as a Seyfert 1. Two position angles have been observed for the main galaxy and no kinematic disturbances are observed.

AM 1427-432. The previously unknown radial velocities of the smaller galaxy seem to be a continuation of the radial velocity distribution of the main galaxy. The rotation curve of the main galaxy has a clear asymmetry with respect to the position corresponding to the maximum emission in the continuum, resulting in the velocity gradient being higher in the direction to the satellite.

$V V 452$. The small satellite galaxy is placed at the end of the tidal arm, with the other arm of the main galaxy being more diffuse and shorter. In this case, we also determined that the two components constitute a physical pair, and also the radial velocity of the smaller galaxy is found to have continuity with the radial velocity curve. PA $160^{\circ}$ : although no major distortions are seen, it is clear that there are more irregularities in the side near the satellite. The observed radial velocity gradient is $30 \mathrm{~km} \mathrm{~s}^{-1} \mathrm{kpc}^{-1}$. $\mathrm{PA} 0^{\circ}$ : the location of the maximum emission in $\mathrm{H} \alpha$ is not coincident with the peak emission in the continuum. The observed radial velocity gradient, in the radius range $0-3 \mathrm{kpc}$ and in the direction to the satellite, is $55 \mathrm{~km} \mathrm{~s}^{-1} \mathrm{kpc}^{-1}$, which is much more pronounced 

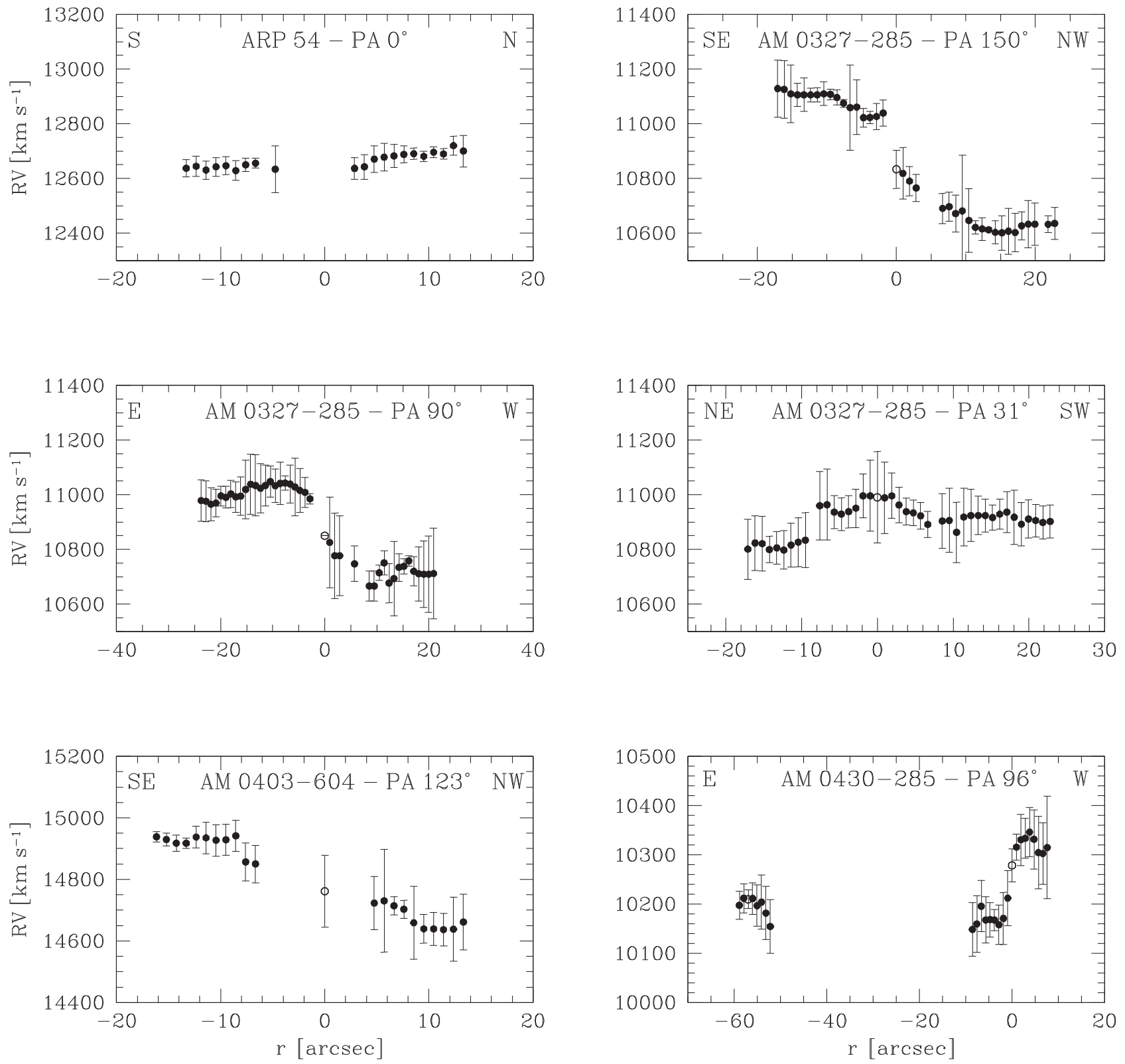

Figure 4. (Continued.)

than in the opposite direction, with a gradient of $15 \mathrm{~km} \mathrm{~s}^{-1} \mathrm{kpc}^{-1}$. The radial velocity of the satellite is similar to that of the nearest measured point in the main galaxy. The gradient in the rigid-body zone is higher on the side of the satellite galaxy. In both observed position angles, the kinematic center does not coincide with the photometric center, the latter being determined by the peak of the continuum emission in the spectrum.

AM 1955-170. No previous radial velocity determinations are known for this system. We performed observations across PA $90^{\circ}$ for the larger galaxy, so it was not possible to confirm whether this is a physical pair, although the morphology suggests it. Unfortunately the $\mathrm{H} \alpha$ emission is strongly contaminated with sky lines so it was not possible to obtain a radial velocity distribution, although the emission in one of the arms is intense enough for us to derive the velocity of the main galaxy, which is $(17180 \pm 30) \mathrm{km} \mathrm{s}^{-1}$.
AM 2058-381. $\mathrm{PA}=9^{\circ}$ (direction along the bar in the main galaxy): from the center (continuum emission peak) and toward the southwest, the curve rises as solid body-like and reaches a relative radial velocity of $195 \mathrm{~km} \mathrm{~s}^{-1}$. In the $\mathrm{NE}$ radius range $0^{\prime \prime}-4^{\prime \prime}$, the curve has a gradient notably shallower than on the southwest side, decreasing by only $20 \mathrm{~km} \mathrm{~s}^{-1}$ with respect to the value corresponding to the central region. After $4^{\prime \prime}$, an abrupt jump of $115 \mathrm{~km} \mathrm{~s}^{-1}$ is observed; thereafter the curve remains nearly constant till $8^{\prime \prime}$ and has another jump of $60 \mathrm{~km} \mathrm{~s}^{-1}$. These jumps could be due to absorption effects (Zasov \& Kopherskov 2003) although the model considered by these authors to explain the jumps is applied in edge-on galaxies. This effect could also be produced by warps. The branch in the curve where these steps are seen is located on the side where the tidal arm connecting to the satellite starts. The kinematic axis coincides with the direction of the bar, therefore the observed rotation curve could also be affected by the gas flows along the bar. 

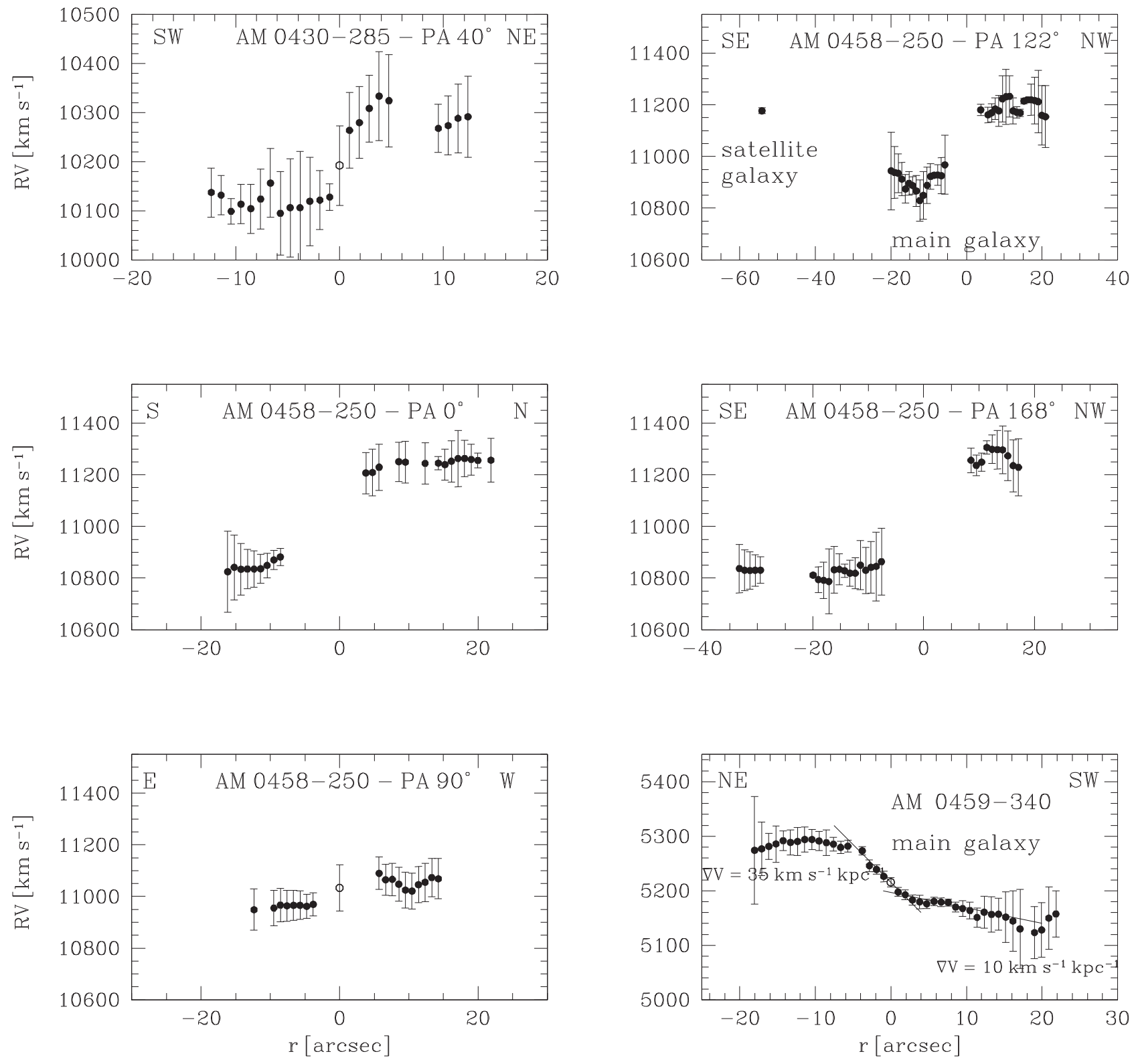

Figure 4. (Continued.)

$A M 2105-332$. For the main galaxy it is only possible to detect $\mathrm{H} \alpha$ emission for the central $5 \mathrm{kpc}$. The amplitude of the rotation curve is $360 \mathrm{~km} \mathrm{~s}^{-1}$. The extent from which we can derive information is too small compared with the extent of the galaxy. Apparently, there is solid-body rotation in the central $2 \mathrm{kpc}$. The observed radial velocity gradient in this zone is large, $144 \mathrm{~km} \mathrm{~s}^{-1} \mathrm{kpc}^{-1}$. No major perturbations are observed in this distribution, although beyond $2 \mathrm{kpc}$ we noted a certain asymmetry. To the southeast the curve remains almost flat, while in the direction to the satellite it rises by $74 \mathrm{~km} \mathrm{~s}^{-1}$.

AM 2256-304. This system could be an extreme case of a high-velocity encounter. The systemic difference in radial velocity between the two galaxies is $1400 \mathrm{~km} \mathrm{~s}^{-1}$. The velocity distribution for the main galaxy is symmetric, presenting oscillations in the flat region of the curve, of $50 \mathrm{~km} \mathrm{~s}^{-1}$ in the north and reaching $8 \mathrm{~km} \mathrm{~s}^{-1}$ in the southern region, where originates the tidal arm that reaches the apparent position of the satellite. These non-circular motions are probably a consequence of the presence of spiral arms. The observed velocity gradient in the solid-body region is $50 \mathrm{~km} \mathrm{~s}^{-1} \mathrm{kpc}^{-1}$. In order to be a bound system, the combined mass of the two components has to be several $10^{12} M_{\odot}$, which matches the approximate total mass of a spiral galaxy dominated by a dark halo. The main galaxy has a diameter of $\sim 20 \mathrm{kpc}$, therefore a high-velocity encounter is a possible scenario.

\subsection{Comparison between Kinematic and Photometric Major Axes}

In Table 3 we list the values corresponding to the position angles of the kinematic major axis of 12 main galaxies of the sample, which were determined from the radial velocity distributions corresponding to two or more position angles. In the same table we list the photometric major axis obtained from the LEDA (these major axes were obtained considering the 25 mag isophote in the $B$-band). The LEDA determinations for the direction of the major axis assign the same weight to the 

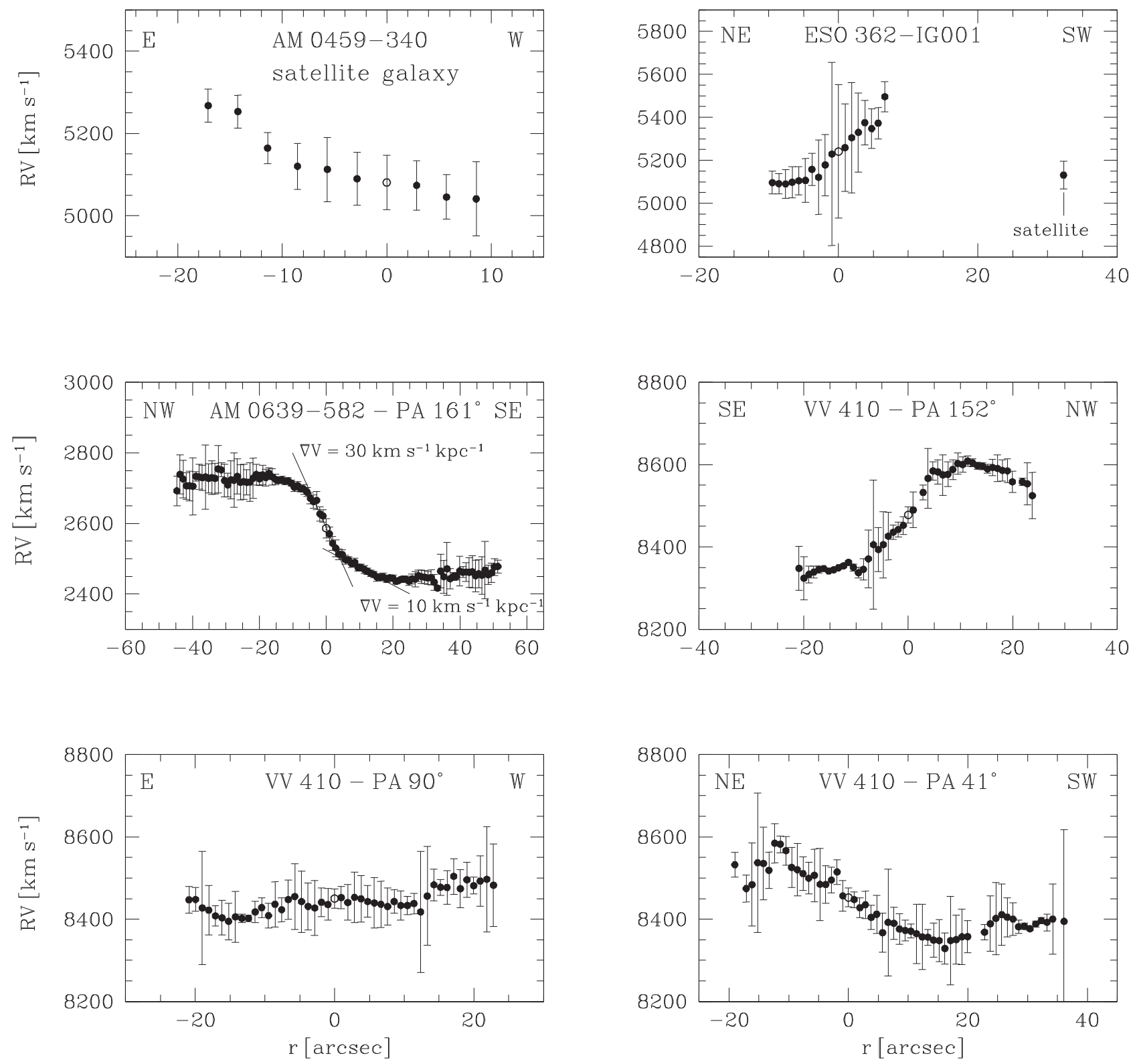

Figure 4. (Continued.)

disk and to other structures as the tidal arm or bridges, so the photometric major axis could be ill defined in several cases. For this reason, we measured the photometric major axis in the $R$ band DSS images, since this band is representative of the more evolved stellar population of the disk. Table 3 includes the position angle of the bars and the absolute values of the differences between the position angles of the major kinematic axis and the major photometric axis. Table 3 also includes the difference in position angle between the bar and the kinematic major axis. No significant discrepancies have been found, with the exception of the larger galaxy of AM 0430-285. However, the value of PA for the major axis is not far from the PA of a small bar-like subsystem of this galaxy. The bar is probably causing the difference between the photometric and kinematic position angles, since the bar could produce non-circular motions. In general, it was found that the PAs of the kinematic and photometric major axes are similar, and if an important deviation is measured, the galaxy has a bar. In conclusion, there is no evidence that in this type of object the interaction is strongly affecting the alignment of the photometric and kinematic major axes, as would be the case in severely warped disk systems.

\subsection{Orbital Plane of the Satellite}

In Table 4 we detail the position angle of the photometric major axis of the main galaxies, the position angle of the center of the satellites, as measured from the centers of the main galaxies, as well as the difference between these values. In Figure 5 we show a histogram of the PA difference. About half of the sample presents a PA difference smaller than $20^{\circ}$, so in a statistical sense this could suggest that satellite galaxies are closer to the plane of the main galaxy. For verification, we have applied a Monte Carlo method, by running a set of simulations for random values of position angle, between $0^{\circ}$ and $90^{\circ}$. Each simulation generates 21 random values in PA difference. For each set we considered its average value, which for the real 

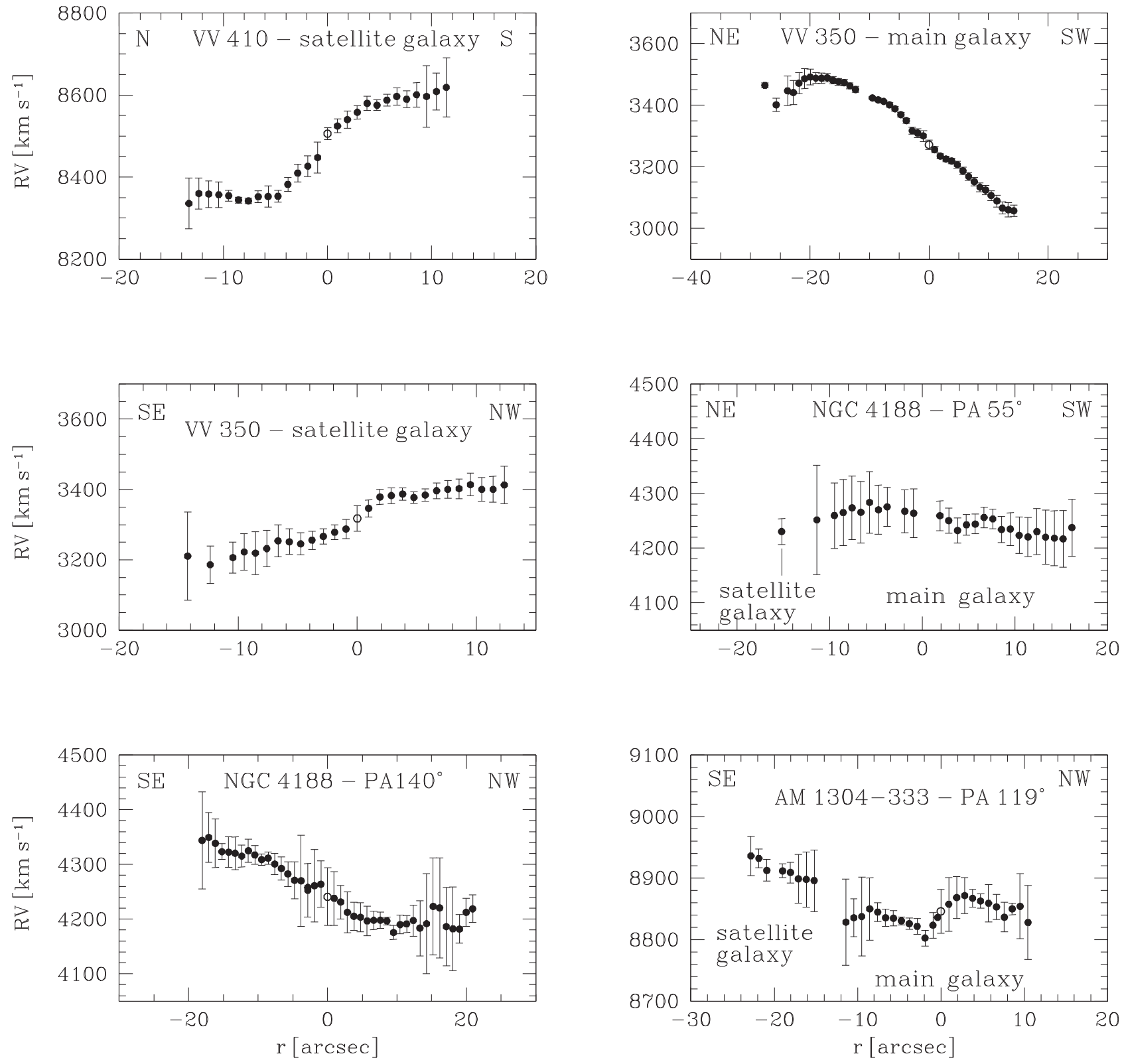

Figure 4. (Continued.)

case is about $28^{\circ}$. After running 1500 random sets, we obtained only three average PA differences under $30^{\circ}: 25^{\circ} .5,28^{\circ} .6$, and $29^{\circ} .5$. This means that the probability of the distribution being random is less than 0.002 , and the orbits of satellite galaxies are significantly closer to the galaxy's equatorial plane during the M51 phase of the interaction.

\subsection{Mass Determinations}

We determined masses in the Keplerian approximation for several members of the present catalog, mainly for the larger components and also for some satellite galaxies. The estimated masses have been obtained for the outermost spectroscopic measurement and considering the maximum amplitude for each velocity distribution. We performed the corresponding correction for inclination of the disk and deviation from the observed position angle with respect to the direction of the major axis. The results are listed in Table 5. At the top of Figure 6 we present the distribution of the masses of the main galaxies. $K s$ band $M / L_{K}$ ratios were calculated using the Keplerian dynamical masses derived from the kinematics, and the integrated $K s$-band luminosities were determined by aperture photometry at the 2MASS $K s$-band images.

For comparison, we considered the galaxies included in the catalog of Karachentsev et al. (2004). This sample consists of 451 Local Volume galaxies (these authors considered objects with distance estimates of $D \lesssim 10 \mathrm{Mpc}$ or radial velocities $V_{\mathrm{LG}}<550 \mathrm{~km} \mathrm{~s}^{-1}$ ). Karachentsev \& Kutkin (2005) obtained $K s$-band luminosities for the mentioned galaxies from 2MASS and studied their relation between mass $/ K s$-band luminosity and $K s$-band luminosity. As we show in Figure 6, the range of $M / L_{K}$ ratios is in very good agreement with the range of values determined by Karachentsev \& Kutkin (2005), who derive the luminosities from 2MASS but calculate the dynamical masses from $\mathrm{HI}$ line widths. However, the M51 sample is located in the same region of the plot of $M / L_{K}$ versus $L_{K}$ in which are 

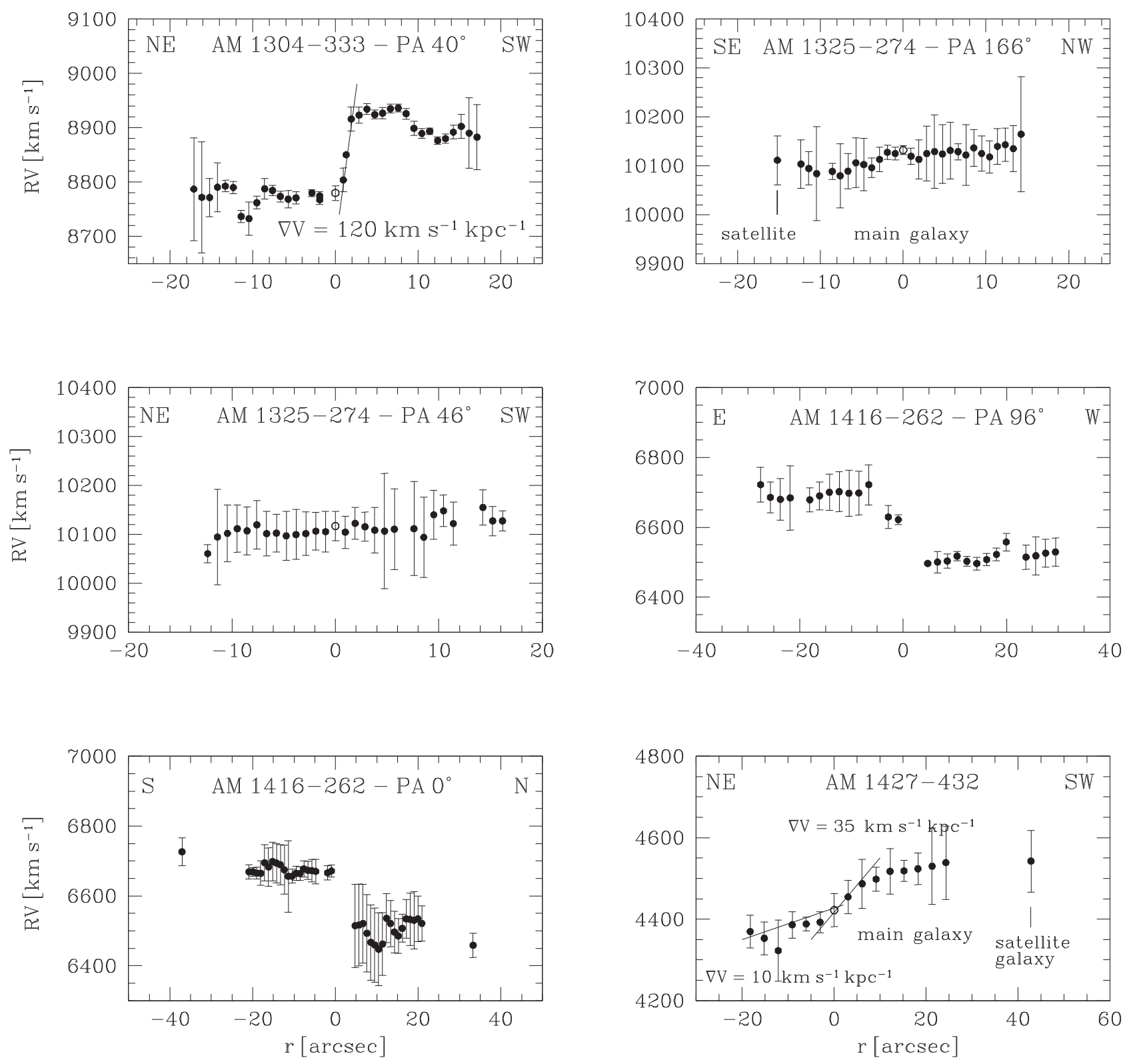

Figure 4. (Continued.)

located the brightest galaxies from Karachentsev et al.'s sample. These authors show that the brightest nearby galaxies detach from the normal distribution of objects, in a subsample with $\log L_{K} \sim 10.5$, comparable with the M51-type galaxy luminosity of about $\log L_{K} \sim 10.8$.

We calculated the $M / L_{K}$ average for the M51-type galaxy sample, $M / L_{K} \sim-0.08$, which is rather below that corresponding to the linear regression of Karachentsev \& Kutkin (2005) at $\log L_{K} \sim 10.8\left(M / L_{K} \sim 0.21\right)$. In the plot, it can be seen that four galaxies have a distinctive low $M / L_{K}$ ratio. We look for evidence of enhanced star formation or kinematic distortion as a possible explanation for the low $M / L_{K}$ observed. These objects with very low $M / L_{K}$ are identified as the main galaxies of AM 1304-333, whose morphology is very distorted and which has a spectrum indicative of enhanced star-forming activity (van den Broek et al. 1991; Günthardt 2009), NGC 633, already classified as a nuclear starburst galaxy (Günthardt 2009), and AM 0430-285, the main galaxy of which has no available spectral data in the literature, and as the satellite galaxy of NGC 633, whose nuclear spectrum is characterized by enhanced star-forming activity (Günthardt 2009). The mean $M / L_{K}$ ratio of the M51-type galaxies excluding these objects is $\sim 0.15$, very close to the value expected from the sample of Karachentsev \& Kutkin (2005).

\subsection{Tully-Fisher Relation}

We studied the Tully-Fisher $(\mathrm{T}-\mathrm{F})$ relation for some of the objects in the present sample. We corrected the maximum velocities for inclination and selected $B$ magnitudes from the LEDA, which are corrected for galactic and internal extinction. The T-F relation for M51-type galaxies in the $B$-band may be fitted by $M(B)=(-2.69 \pm 0.78) \times\left(\log V_{\max }\right)-(14.86 \pm 1.60)$. In Figure 7, we compared our fitting for M51-type galaxies with the T-F relation obtained by Kudrya \& Karachentseva (2012) for 169 isolated edge-on galaxies (these objects are listed in the 2MIG catalog of isolated galaxies; Karachentseva et al. 2010). The T-F 

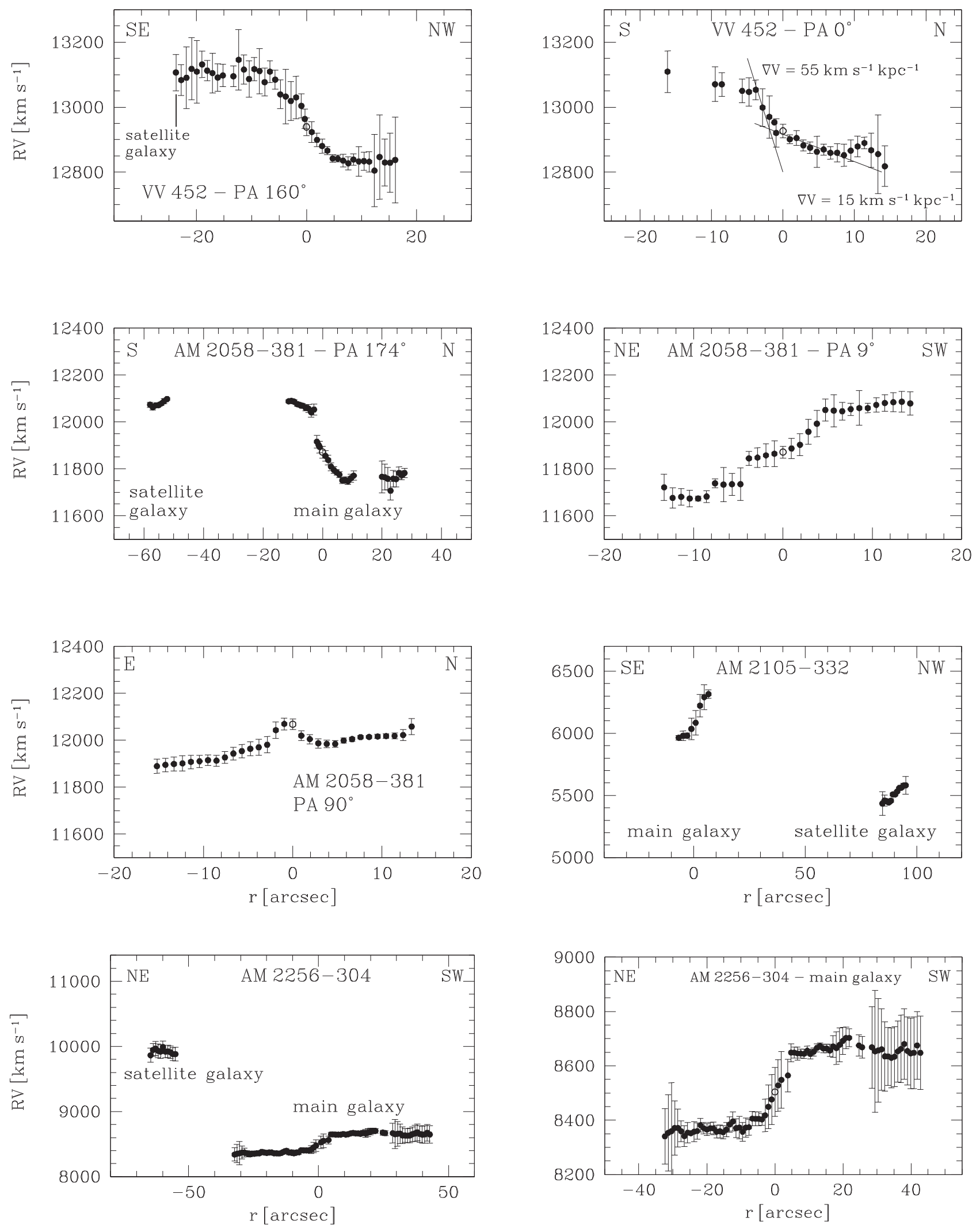

Figure 4. (Continued.)

fitting for M51-type galaxies is flatter than the relation corresponding to isolated galaxies. As can be observed in Figure 7 (upper left) the slope corresponding to the fitting for member galaxies of M51-type systems is also flatter than the slope of the classic T-F relation (Pierce \& Tully 1992; Tully et al. 1998; Tully \& Pierce 2000). Reshetnikov \& Klimanov (2003, hereafter RK) also obtain, for a sample of M51-type galaxies, a T-F relation flatter than the relation corresponding to nearby galaxies. If we 
Table 2

Systemic Heliocentric Radial Velocities

\begin{tabular}{|c|c|c|c|c|c|}
\hline Object & $V_{\text {main }}\left(\mathrm{km} \mathrm{s}^{-1}\right)$ & $V_{\text {main }}\left(\mathrm{km} \mathrm{s}^{-1}\right)$ & $V_{\text {sat }}\left(\mathrm{km} \mathrm{s}^{-1}\right)$ & $V_{\text {sat }}\left(\mathrm{km} \mathrm{s}^{-1}\right)$ & $\delta V\left(\mathrm{~km} \mathrm{~s}^{-1}\right)$ \\
\hline NGC 633 & $5098(5)$ & 5103 & $5168(10)$ & $\cdots$ & 70 \\
\hline ARP 54 & $12628(30)$ & 12666 & $\cdots$ & $\cdots$ & $60^{\mathrm{a}}$ \\
\hline AM $0327-285$ & $10833(60)$ & 10853 & $\cdots$ & $\cdots$ & $94^{\mathrm{b}}$ \\
\hline AM 0430-285 & $10279(30)$ & $\ldots$ & $10209(25)$ & $\ldots$ & 70 \\
\hline AM $0458-250$ & $11018(20)$ & 11043 & 11177 (12) & $\ldots$ & 159 \\
\hline AM 0459-340 & $5215(8)$ & 5209 & $5081(30)$ & $\ldots$ & 134 \\
\hline VV 410 & $8478(20)$ & 8473 & 8506 (14) & $\cdots$ & 28 \\
\hline VV 350 & $3272(15)$ & $\ldots$ & $3318(35)$ & $\ldots$ & 46 \\
\hline NGC 4188 & $4241(50)$ & 4261 & $4230(20)$ & $\ldots$ & 11 \\
\hline AM $1304-333$ & 8846 (10) & 8852 & $8910(15)$ & $\ldots$ & 64 \\
\hline AM $1325-274$ & $10131(10)$ & $\ldots$ & $10111(35)$ & $\ldots$ & 20 \\
\hline AM 1416-262 & & 6600 & $\ldots$ & $\ldots$ & $139^{\mathrm{b}}$ \\
\hline AM $2105-332$ & $\ldots$ & $6147(15)$ & $\ldots$ & $5512(7)$ & 591 \\
\hline AM 2256-304 & $8504(70)$ & 8507 & $9920(50)$ & $\ldots$ & 1416 \\
\hline
\end{tabular}

Notes. Column (1): object name. Columns (2) and (4): systemic radial velocities obtained at the position of the peak of the continuum emission. Columns (3) and (5): systemic radial velocities obtained from the center of symmetry of the radial velocity curve. Column (6) modulus of the difference between the systemic radial velocities of main and satellite galaxies. Estimated uncertainties $(2 \sigma)$ are listed between brackets, and are derived from the empirical expression for $\sigma$ from Keel (2004).

${ }^{a}$ Difference in radial velocity obtained from CASLEO spectrophotometric observations.

${ }^{\mathrm{b}}$ Difference in $\mathrm{r}$ adial velocity extracted from NED data.

Table 3

DATA on Kinematic and Photometric Major Axes for Main Galaxies

\begin{tabular}{|c|c|c|c|c|c|c|c|}
\hline Object & PA (KMA) (deg) & PA (LEDA) (deg) & $|\mathrm{C} 3-\mathrm{C} 2|(\mathrm{deg})$ & PA (R-DSS) (deg) & $|\mathrm{C} 5-\mathrm{C} 2|(\mathrm{deg})$ & PA (bar) (deg) & $|\mathrm{C} 7-\mathrm{C} 2|(\mathrm{deg})$ \\
\hline VV 410 & $175 \pm 15$ & 8 & 13 & $\cdots$ & $\cdots$ & $152 \pm 3$ & $23 \pm 18$ \\
\hline NGC 633 & $135 \pm 10$ & $\cdots$ & $\cdots$ & $138 \pm 10$ & $3 \pm 20$ & $\cdots$ & $\cdots$ \\
\hline ARP 54 & $130 \pm 15$ & 112 & 18 & $123 \pm 5$ & $7 \pm 20$ & $\ldots$ & $\cdots$ \\
\hline AM 0458-250 & $165 \pm 10$ & 167 & 2 & $168 \pm 3$ & $10 \pm 3$ & $\cdots$ & $\cdots$ \\
\hline AM $0327-285$ & $150 \pm 15$ & 167 & 17 & $\ldots$ & $\cdots$ & $\cdots$ & \\
\hline AM $0430-285^{*}$ & $170 \pm 20$ & 79 & 81 & $50 \pm 5$ & $60 \pm 5$ & $136 \pm 3$ & $34 \pm 23$ \\
\hline VV $452^{*}$ & $165 \pm 20$ & 164 & 1 & $150 \pm 5$ & $15 \pm 25$ & $\cdots$ & $\cdots$ \\
\hline NGC 4188* & $140 \pm 20$ & $\cdots$ & $\cdots$ & $145 \pm 5$ & $5 \pm 25$ & $\cdots$ & $\cdots$ \\
\hline AM $1325-274^{*}$ & $100 \pm 25$ & $\cdots$ & $\cdots$ & $\cdots$ & $\cdots$ & $\cdots$ & $\cdots$ \\
\hline
\end{tabular}

Note. Column (1): object name (those objects for which their kinematic major axis has been determined from only two position angles are highlighted with an asterisk). Column (2): position angle of the kinematic major axis. Column (3): position angle of the photometric major axis, extracted from LEDA. Column (4): modulus of the difference between the corresponding values of columns (2) and (3). Column (5): our own determinations of photometric major axis, from R-DSS images. Column (6): modulus of the difference between values corresponding to columns (5) and (2). Column (7): position angle of the bar subsystem. Column (8): modulus of the difference between values corresponding to columns (7) and (2).

add the data from RK to our own data in the $\mathrm{T}-\mathrm{F}$ relation, the same trend is observed and may be fitted by $M(B)=$ $(-3.20 \pm 0.64) \times\left(\log V_{\max }\right)-(13.56 \pm 1.11)$. Comparing our sample with the data of RK, there are only three objects in common, the main and satellite galaxies of VV 350 and the main galaxy of VV 452. In Figure 7 (upper right) the galaxies of both samples are included although, considering that the only source of the RK data is their Figure 4, that is, we cannot distinguish which object corresponds to each TF point in the plot of RK, we decided not to include the data corresponding to VV 350 and VV 452 of our sample. The fitting of our sample is flatter than the fitting of RK, because their sample includes two objects of lower masses and luminosities, making their fit steeper than ours. As may be seen, the brightest galaxies are placed near the standard relation, 
Table 4

Relative Positions of Satellite Galaxies

\begin{tabular}{lccc}
\hline \hline & Major Axis & & \\
Object & PA (deg) & Satellite PA (deg) & $\Delta($ PA) (deg) \\
\hline NGC 633 & 168 & 8 & 20 \\
ARP 54 & 90 & 76 & 14 \\
AM 0327-285 & 150 & 150 & 0 \\
AM 0403-604 & 117 & 63 & 54 \\
AM 0430-285 & 103 & 99 & 4 \\
AM 0458-250 & 158 & 123 & 35 \\
AM 0459-340 & 20 & 162 & 38 \\
ESO 362-IG001 & 40 & 30 & 10 \\
AM 0639-582 & 168 & 8 & 20 \\
VV 410 & 163 & 176 & 13 \\
VV 350 & 51 & 48 & 3 \\
NGC 4188 & 140 & 58 & 82 \\
AM 1304-333 & 40 & 122 & 82 \\
AM 1325-274 & 139 & 170 & 31 \\
AM 1416-262 & 6 & 97 & 89 \\
AM 1427-432 & 7 & 43 & 36 \\
VV 452 & 150 & 159 & 9 \\
AM 1955-170 & 0 & 31 & 31 \\
AM 2058-381 & 3 & 171 & 12 \\
AM 2105-332 & 135 & 146 & 11 \\
AM 2256-304 & 11 & 11 & 0 \\
\hline
\end{tabular}

Note. Column (1): object name; column (2): position angle of the photometric major axis of the main galaxy; column (3): position angle of the center of the satellite galaxy, as measured from the center of the main galaxy; column (4): modulus of the difference in position angle between the values of columns (2) and (3).

which is compatible with the fact that, for the most massive systems, the T-F relation does not depend on the environment (Barton et al. 2001). The less massive galaxies, members of M51type systems, have a higher luminosity than would correspond to a standard $\mathrm{T}-\mathrm{F}$ relation. The average $B$-band flux ratio is $\sim 4$ between the luminosity of M51-type galaxies with velocities lower than $\log \left(V_{\max }\right)=2.1$ and the luminosity expected from the T-F fitting of the isolated galaxies of the sample of Kudrya \& Karachentseva (2012). Following this line of thought, we add a study of the $\mathrm{T}-\mathrm{F}$ relation in the $K s$-band, since this band may be less affected by recent star formation. The result (bottom of Figure 7) is also a flatter slope when compared with the fitting of 182 isolated edge-on galaxies of Kudrya \& Karachentseva (2012). For comparison, the T-F relation found by Torres-Flores et al. (2011; GHASP spiral galaxies) is also shown in Figure 7, as well as the fitting of Masters et al. (2008) for 2MASS galaxies.

The average $K s$-band flux ratio is $\sim 6$ between M51-type galaxies with radial velocities $\operatorname{lower}$ than $\log \left(V_{\max }\right)=2.1$ and the $\mathrm{T}-\mathrm{F}$ fitting in the $K s$-band for the sample of Kudrya \& Karachentseva (2012).

A similar situation is observed in the study of members of Hickson compact groups of galaxies (Torres-Flores et al. 2013), where a few low-mass outlier objects deviate from the standard $\mathrm{T}-\mathrm{F}$ relation for field galaxies. We noticed that these outliers occupy the same region in the plot of the T-F relation as the low-mass M51-type galaxies. This happens for both $B$ band and $K s$-band T-F relations. According to Torres-Flores et al. (2013), the deviations from the standard relation could be due to a combination of a flux increment generated by the enhancement of star-forming events with the uncertainties in the determination of the kinematic widths due to kinematic

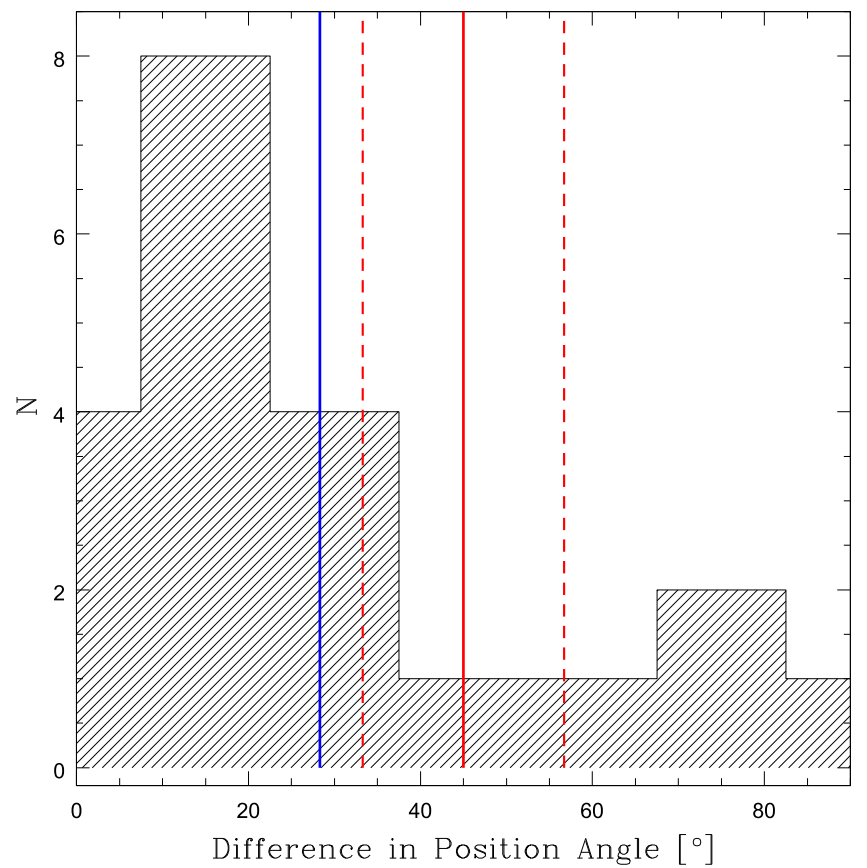

Figure 5. Histogram distribution of the difference between the position angle of the main galaxy's major axis and the position angle of the segment that connects the centers of the main and satellite galaxies. There is clear excess of companions located toward the major axis of the main galaxy, with the observed average marked with a blue line in the plot. This indicates that the systems are in a phase of the interaction in which the orbit has a large projection on the plane of the main galaxy. A selection effect of M51 companions located nearer to the galaxy's major axis can be discarded because there is no correlation with the inclination of the system. After 1500 Monte Carlo simulation runs of sets of position angle, the average of the mean differences of the simulated sets (of 21 objects each) is drawn with a red solid line, and the two dashed lines correspond to the limit of three times the standard deviation $( \pm 3 \sigma)$. The distribution of PA difference in the observed sample is clearly not random.

distortions induced by gravitational interactions. Torres-Flores et al. (2013) observe for some outliers a larger deviation in the $K s$-band than in the $B$-band. They notice that, according to Maraston (1998), if thermally pulsating asymptotic giant branch (TP-AGB) stars are considered in models of stellar population synthesis, they may make an important contribution in the $K$-band. In particular, Maraston (1998) found that this effect is significant for an evolved burst with an age of about 700 Myr.

Another study of objects that are suffering gravitational interactions is that of Barton et al. (2001), who determine the $\mathrm{T}-\mathrm{F}$ relation for galaxy pairs. They also observe for low-mass objects an excess in the $B$-band flux over that expected for standard $\mathrm{T}-\mathrm{F}$ relations. These authors determined that in extreme cases the deviation from the standard $\mathrm{T}-\mathrm{F}$ relation may be explained by uncertainties in inclination, in which cases long tidal tails should be observed. Since M51-type galaxies in general present long tidal tails, the effects due to indetermination in inclination in our sample could be larger than those in the sample of Barton et al. (2001) also proposed that another possible explanation for the outliers is dissipative effects on the rotation curve (gas infall and non-uniform or truncated gas emission).

Our M51-type galaxy sample includes low-mass objects with asymmetric curves (e.g., AM 0459-340, AM 1304-333, NGC 633), truncated rotation curves (NGC 633 satellite, 
Table 5

Keplerian Mass Estimations

\begin{tabular}{|c|c|c|c|c|c|}
\hline Object & Inclination (deg) & PA (obs.) (deg) & PA Major Axis (deg) & $R(\mathrm{kpc})$ & $\overline{\text { Mass }\left(10^{11} M_{\odot}\right)}$ \\
\hline AM 0639-582 & 65 & 161 & 174 & 8 & $0.40 \pm 0.05$ \\
\hline VV 410 (A) & 51 & 152 & 170 & 14 & $1.4 \pm 0.2$ \\
\hline VV 410 (B) & 68 & 0 & 0 & 7 & $0.34 \pm 0.06$ \\
\hline NGC 633 (A) & 49 & 136 & 136 & 6 & $0.20 \pm 0.09$ \\
\hline NGC 633 (B) & 65 & 0 & 0 & 2 & $0.020 \pm 0.009$ \\
\hline ARP 54 & 53 & 90 & 90 & 25 & $3.5 \pm 0.2$ \\
\hline AM 2256-304 & 58 & 11 & 11 & 25 & $2.5 \pm 0.3$ \\
\hline AM 2058-381 & 60 & 9 & 9 & 11 & $2.0 \pm 0.2$ \\
\hline AM 0459-340 (A) & 65 & 20 & 28 & 6 & $0.15 \pm 0.09$ \\
\hline AM 0459-340 (B) & 63 & 0 & 0 & 6 & $0.010 \pm 0.005$ \\
\hline AM 0458-250 & 43 & 168 & 152 & 16 & $4.6 \pm 2.0$ \\
\hline AM 0430-285 & 50 & 40 & 40 & 10 & $0.15 \pm 0.09$ \\
\hline AM $1416-262$ & 45 & 0 & 6 & 16 & $1.2 \pm 0.3$ \\
\hline AM 0403-604 & 46 & 123 & 117 & 15 & $1.7 \pm 0.3$ \\
\hline AM 1304-333 & 60 & 40 & 40 & 10 & $0.10 \pm 0.09$ \\
\hline ESO 362-IG001 & 51 & 29 & 29 & 3 & $0.30 \pm 0.03$ \\
\hline VV 452 & 50 & 160 & 150 & 20 & $1.9 \pm 0.3$ \\
\hline VV 350 (A) & 73 & 55 & 55 & 6 & $0.7 \pm 0.1$ \\
\hline VV 350 (B) & 66 & 126 & 96 & 4 & $0.15 \pm 0.03$ \\
\hline AM $0327-285$ & 44 & 150 & 150 & 14 & $4.7 \pm 2.0$ \\
\hline
\end{tabular}

Note. Column (1): object name; column (2): inclination of main galaxy (angle between the disk plane and the line of sight); column (3): observed position angle; column (4): position angle of major axis; column (5): the listed value corresponds to the outermost position for which a spectroscopic measurement has been made; column (6): Keplerian masses (corrected for inclination of the disk and deviation of the observed position angle with respect to the direction of the major axis).

NGC 4188), and star-forming enhancement (AM 0459-340, main and satellite galaxies of NGC 633, AM 1304-333). In addition, we must take into account that in objects with well marked signs of gravitational interactions the determination of the major axis is more tricky, possibly resulting in an underestimation of the amplitude of the rotation curve. A next step on this subject could be to perform a study using 3D spectroscopic techniques. Few low-mass M51 objects have been studied, so it would also be desirable to add observations of less massive systems.

\subsection{Synthetic Radial Velocity Distribution}

For future numerical simulations of the gravitational encounter between main and satellite galaxies (components of a typical M51-type system), it is necessary to consider a representative radial velocity curve of the larger component. One possible approach is to derive a synthetic curve from the available radial velocity data for a set of main galaxies. For this purpose, we selected from our catalog the radial distributions along the position angle of the major axis for 13 systems (data_a, Table 6), retaining the systems that present less ambiguity in the determination of the position angle of the major axis and the inclination. The radial velocity data have been deprojected, considering the inclination of the main galaxy. Being more restrictive, we excluded some systems and built two other data sets, data_b (11 objects) and data_c (9 objects), the last set of data being the one with more constrained parameters, so this last set of radial velocities contains the most "trusty" data values. The data have been normalized, in both radial velocity and position, by dividing by the peak value in radial velocity, and in the case of the position, dividing by the disk extent of the main component in the RDSS band.
For fitting the data, we have used the software "TableCurve." The functions ${ }^{8}$ that resulted in a best fitting for each set of data are: "Asymmetric Double Gaussian Cumulative" (ADC) for data_a, "Modified Gaussian" (GaussMod) for data_b, and "Symmetric Double Gaussian Cumulative" (SDC) for data_c. In Table 7 we list the coefficients of the functions that fitted the data for each set (Figure 8). In the same table we include the standard deviation $(\sigma)$. Also, for each data set, and as a simple visual test of the fitting, we divided the range of normalized radial distance into different bins, obtaining an average value of normalized radial velocity for each bin. The functions obtained with the mentioned software provided a good fitting to the average values. The errors associated with the average values correspond to the standard deviation and are plotted in Figure 8. As can be seen in Figure $8(\mathrm{~d})$, there is no significant difference between the fittings of the three sets of data.

As a different approach, we have built a representative gravitational potential of the spiral galaxies corresponding to set "c." This spiral galaxy model consists of a disk described by an exponential law (Freeman 1970), plus a dark halo represented by an NFW mass distribution (Navarro et al. 1996). The choice of these potentials corresponds to the description of Barnes \& Hibbard (2009) for the numerical modeling of interacting disk galaxies. Considering that we have obtained radial velocities from long-slit spectroscopy (with half-arcsecond uncertainties in the position of the galaxy's center), the galactic centers are affected by important interstellar extinction (underestimated radial velocities), and considering the seeing of the observations, we conclude that the central radial velocities have significant uncertainties. Therefore, the resolution obtained for small radii is not adequate to

\footnotetext{
8 A description of the functions used in this fitting process, as well as their coefficients $(a, b, c, d, e$, and $f)$ may be found in http://systat.co.kr/products/ TableCurve2D/help/1041.html.
} 

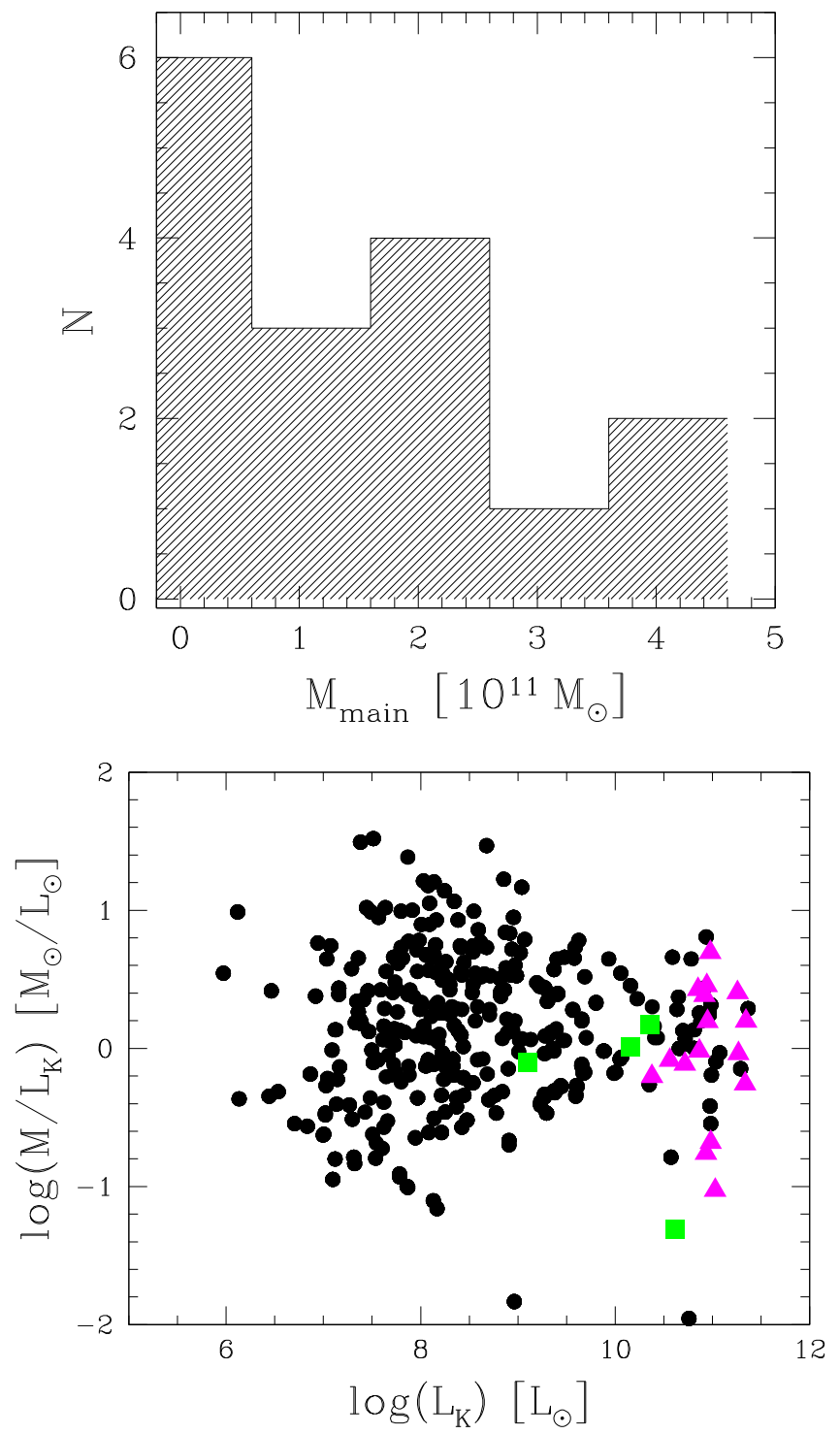

Figure 6. Top: histogram distribution of estimated Keplerian masses, derived for the main galaxies of the M51-type systems. Bottom: in this plot of $\log \left(M / L_{K}\right)$ vs. $\left(L_{K}\right)$ are included the values corresponding to our sample of M51-type galaxies: magenta triangles for main galaxies and green squares for satellite galaxies, while black circles show the values corresponding to the galaxies of Karanchentsev et al.'s catalog (see Karachentsev et al. 2004 and Figure 6 of Karachentsev \& Kutkin 2005). The $K$-band luminosity is in units of the $K$-band solar luminosity.

properly trace the mass of the bulge component. Consequently, we have considered only the disk and halo components from the dynamic model proposed by Barnes \& Hibbard (2009). In order to resolve the well known disk-halo conspiracy in the description of rotation curves, we have assumed the parameter relation proposed by Barnes \& Hibbard (2009) for both components. The best description for the circular velocities, according to the adopted model, is shown in Figure 9(a). Assuming for the sample set "c" average values of the maximum circular velocity and maximum radius of $190 \mathrm{~km} \mathrm{~s}^{-1}$ and $11.75 \mathrm{kpc}$ respectively, the total mass of the disk is $M_{\text {disk }}=(4.75 \pm 0.35) \times 10^{10} M_{\odot}$ with a scale radius of $a_{d}=(3.5 \pm 0.2) \mathrm{kpc}$, while the halo mass inside the scale radius is $M_{\text {halo }}\left(a_{h}\right)=(4.0 \pm 1.1) \times 10^{10} M_{\odot}$ with a scale radius of $a_{h}=(10.5 \pm 0.3) \mathrm{kpc}$. We listed the latter parameters in Table 7, as well as the standard deviation of fitting. In order to take into
Table 6

Selected Data Used for Deriving the Synthetic Curve

\begin{tabular}{lccc}
\hline \hline Object & data_a & data_b & data_c \\
\hline NGC 633 & $\checkmark$ & $\checkmark$ & $\checkmark$ \\
ARP 54 & $\checkmark$ & $\checkmark$ & $\checkmark$ \\
AM 0327-285 & $\checkmark$ & $\checkmark$ & $\ldots$ \\
AM 0403-604 & $\checkmark$ & $\ldots$ & $\ldots$ \\
AM 0458-250 & $\checkmark$ & $\checkmark$ & $\checkmark$ \\
AM 0459-340 & $\checkmark$ & $\checkmark$ & $\checkmark$ \\
AM 0639-582 & $\checkmark$ & $\checkmark$ & $\checkmark$ \\
VV 410 & $\checkmark$ & $\checkmark$ & $\ldots$ \\
VV 350 & $\checkmark$ & $\checkmark$ & $\checkmark$ \\
NGC 4188 & $\checkmark$ & $\ldots$ & $\ldots$ \\
VV 452 & $\checkmark$ & $\checkmark$ & $\checkmark$ \\
AM 2058-381 & $\checkmark$ & $\checkmark$ & $\checkmark$ \\
AM 2256-304 & $\checkmark$ & $\checkmark$ & $\checkmark$ \\
\hline
\end{tabular}

Note. Column (1): object designation; columns (2)-(4): selected objects used for three data sets_-data_a, data_b, and data_c_-from which synthetic curves are derived.

account the observational uncertainties in the central region, we have performed a second fitting of the data just beyond $R=1.5 \mathrm{kpc}$, including a spherical potential (Hernquist 1990), as proposed by Barnes \& Hibbard (2009), which contains $5 \%$ of the total mass and has a scale radius of $24 \%$ of that of the disk component (Barnes \& Hibbard 2009). In this case, the fitting parameters were allowed to vary from the relations of Barnes \& Hibbard (2009) by less than $25 \%$ in mass and less than $10 \%$ in scale radius. The mass model parameters including a bulge component are the following: $M_{\text {bulge }}=1.12 \times 10^{10} M_{\odot}$, $a_{b}=0.85 \mathrm{kpc} ; \quad M_{\text {disk }}=$ $3.5 \times 10^{10} M_{\odot}, a_{d}=3.5 \mathrm{kpc} ; M_{\text {halo }}\left(a_{h}\right)=3.8 \times 10^{10} M_{\odot}, a_{h}=$ $9.5 \mathrm{kpc}$. Thereby, a comparison between this synthetic rotation curve and higher-resolution rotation curves is possible. This synthetic rotation curve serves as a model starting point for numerical simulations of M51-type galaxies.

In order to further take into account the dispersion of observational data, we have determined two rotation curve envelopes that enclosed the individual rotation curves of set "c" (Figure 9(c)). The envelope curves keep the same scale parameters but differ in mass by $50 \%$. Another useful description for these rotation velocities is a simple linear fitting function that could be easily compared with other samples. The linear fitting consists of two straight lines, that is, one that represents the central rigid rotation $(R<2 \mathrm{kpc})$ and another that represents the flat region of the rotation curve $(R>3 \mathrm{kpc})$. In Figure $9(\mathrm{~d})$ we show the fitting of both linear functions. The central region has a radial velocity gradient of $68 \mathrm{~km} \mathrm{~s}^{-1} \mathrm{kpc}^{-1}$ while the flat region shows a slight increase with radius of $1.1 \mathrm{~km} \mathrm{~s}^{-1} \mathrm{kpc}^{-1}$ (this slight slope is consistent with the previous synthetic description that includes a largescale halo component).

\section{CONCLUSIONS}

From the present kinematic sample consisting of 21 interacting M51-type galaxy systems, we found that the radial velocity distributions present asymmetries in about half of the studied main galaxies. Typical examples are AM 0459-340, VV 410, and VV 452. In some systems a detailed scrutiny reveals subtle asymmetries: at first sight the curve of AM 0639- 

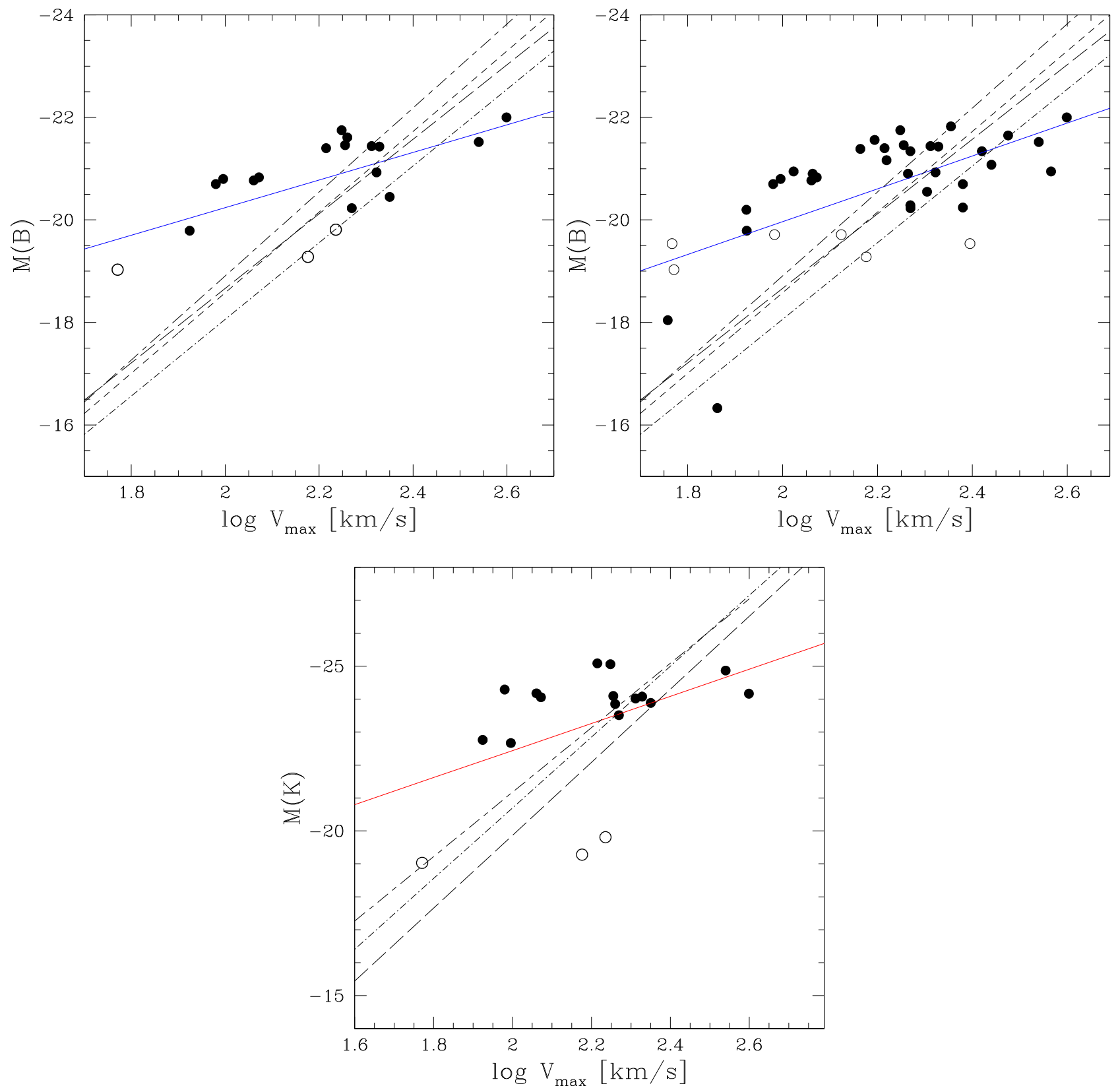

Figure 7. Upper left: Tully-Fisher relation for the sample galaxies in the $B$-band. The blue solid line shows the fit to the data. Also plotted, for comparison, are the standard Tully-Fisher relation of Tully et al. (1998, short-dashed line) and the relations of Tully \& Pierce (2000, long-dashed line), Pierce \& Tully (1992, dasheddotted line), and Kudrya \& Karachentseva (2012, short-dashed-long-dashed line). Upper right: Tully-Fisher relation in the B-band, including the galaxies of the present sample and the objects from Reshetnikov \& Klimanov (2003). The blue solid line shows the fit to the data. The comparison fitting lines are the same as those in the upper left plot. Bottom: $K$-band Tully-Fisher relation corresponding to the sample of the present work. The solid line shows the best fit to the data, and for comparison we also plotted the relationships found by Kudrya \& Karachentseva (2012, short-dashed-long-dashed line), Torres-Flores et al. (2011, long-dashed line), and Masters et al. (2008, dashed-dotted line). In all the plots filled circles correspond to the main galaxies and open circles to satellite galaxies.

582 is normal; however, the observed radial velocity gradients are different on the two sides of the distribution, taking as reference the position corresponding to the red continuum emission peak in the spectrum.

Only AM 1304-333 has a very distorted radial velocity curve. We also observe an important morphological perturbation, which may be the result of an ongoing multiple merger.

For almost all the main galaxies that belong to the present sample, the observed perturbations are not as significant as those involved in mergers. In fact we found only small or moderate differences between the photometric and kinematic major axes of the main galaxies. In systems with a low ratio between the masses of the satellite and the main galaxy, the systematic velocities of the satellites are a continuation of the velocity curve of the larger galaxy, which agrees with the results obtained by Reshetnikov \& Klimanov (2003): the relative velocity of the satellite is approximately equal to the velocity in the outer parts of the disk of the main galaxy.

M51-type galaxies have average luminosities $\left(\log L_{K} \sim 10.5\right)$ that are comparable to the luminosities of the brightest objects in the nearby galaxy sample (451 objects) of Karachentsev \& Kutkin (2005). This may be due to a selection bias (we are 
Table 7

Coefficients of the Fittings to the Synthetic Rotation Curves

\begin{tabular}{|c|c|c|c|c|c|c|c|c|c|c|c|c|c|c|}
\hline & Function & $a$ & $\bar{b}$ & $c$ & $d$ & $e$ & $f$ & $r^{2}$ & $\sigma$ & $M_{\text {disk }}\left(10^{10} M_{\odot}\right)$ & $a_{d}(\mathrm{kpc})$ & $M_{\text {halo }}\left(10^{10} M_{\odot}\right)$ & $a_{h}(\mathrm{kpc})$ & $\sigma\left(\mathrm{km} \mathrm{s}^{-1}\right)$ \\
\hline data_a & $\mathrm{ADC}$ & 3.673 & 0.051 & 0.086 & 0.179 & 50.410 & -0.871 & 0.76 & 0.15 & $\cdots$ & $\cdots$ & $\cdots$ & $\cdots$ & $\cdots$ \\
\hline data_b & GaussMod & 1.508 & 8641.187 & 8641.1024 & 70015.656 & -0.549 & $\cdots$ & 0.82 & 0.12 & $\cdots$ & $\cdots$ & $\cdots$ & $\cdots$ & $\cdots$ \\
\hline data_c & SDC & 3.287 & 1.690 & 3.625 & 0.220 & -2.327 & $\cdots$ & 0.85 & 0.10 & $4.75 \pm 0.35$ & $3.5 \pm 0.2$ & $4.0 \pm 1.1$ & $10.5 \pm 0.3$ & 19 \\
\hline
\end{tabular}

Note. Column (1): data set used for the fitting function; column (2): fitting function; columns (3)-(8): coefficients $a, b, c, d, e$, and $f$ of the corresponding fitting functions, which are listed in the Appendix; column (9): coefficient of determination; column (10): standard deviation of the fitting; column (11): disk mass derived by using the potentials proposed by the simulation of Barnes \& Hibbard (2009); column (12): disk scale radius; column (13): halo mass; column (14): halo scale radius; column (15): standard deviation of the fitting.
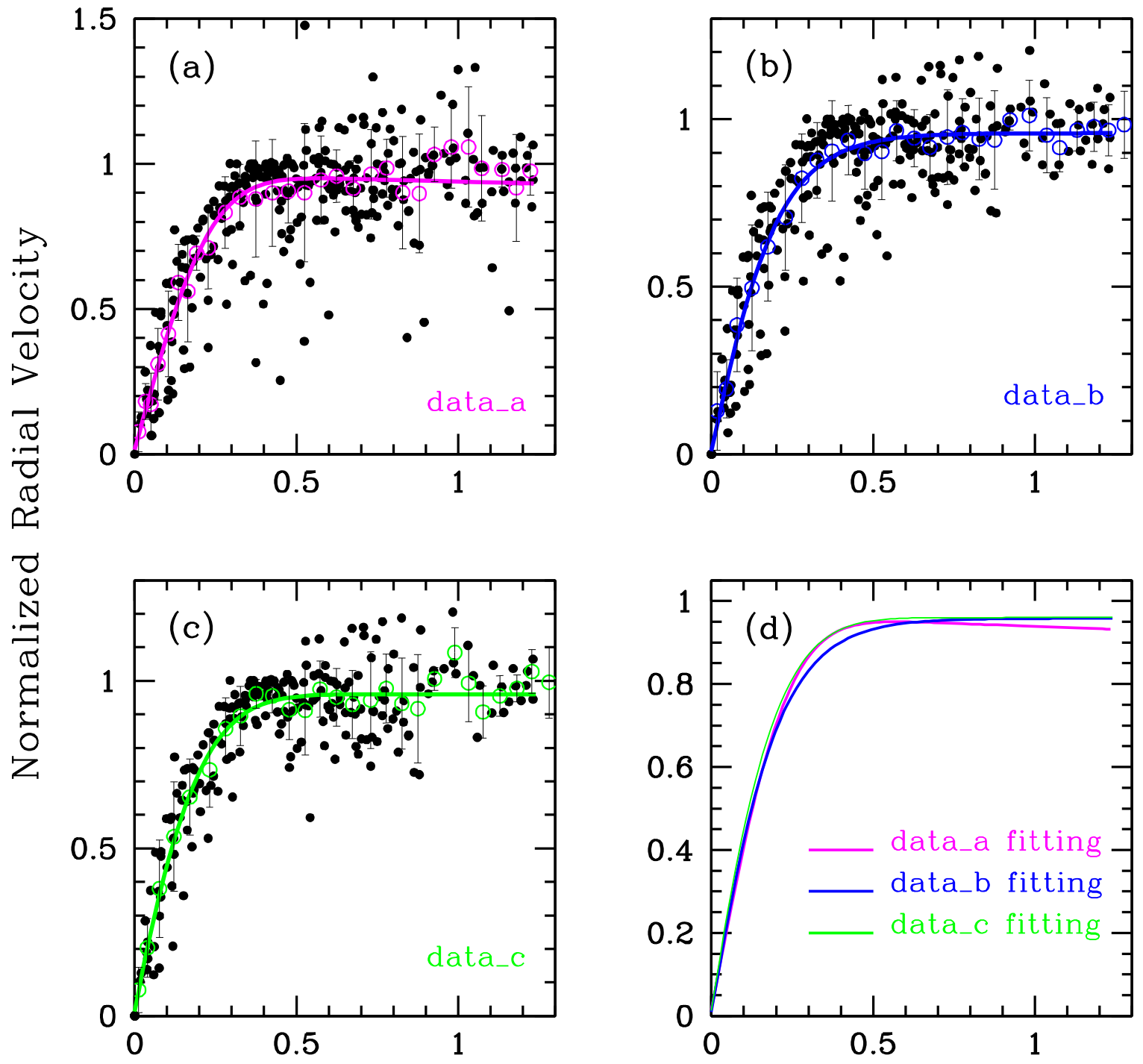

\section{Normalized Radial Distance}

Figure 8. Panels (a)-(c) show the radial velocity data with their corresponding fitting, for the three sets considered: data_a, data_b, and data_c, respectively. Panel (d) shows the fitting for the three sets of data.

detecting only the brightest M51 galaxies), but the relevance of this bias seems diminished by the fact that only the prototype M51 (NGC 5194/95) in Karachentsev's sample matches the type of objects considered here, and that this prototype system is also a luminous one. The brightness of the galaxy in the $K$-band could be raised by an enhancement of the young stellar population in these galaxies, shifting the location of M51 galaxies toward lowered $M / L_{K}$ ratios in the plot. This effect is seen for some less massive objects in the $K s$-band Tully-Fisher relation. Therefore the M51 phenomenon happens in intrinsically massive bright spiral galaxies (such as the case of M51 itself), which nevertheless have quite normal $M / L_{K}$ ratios. 

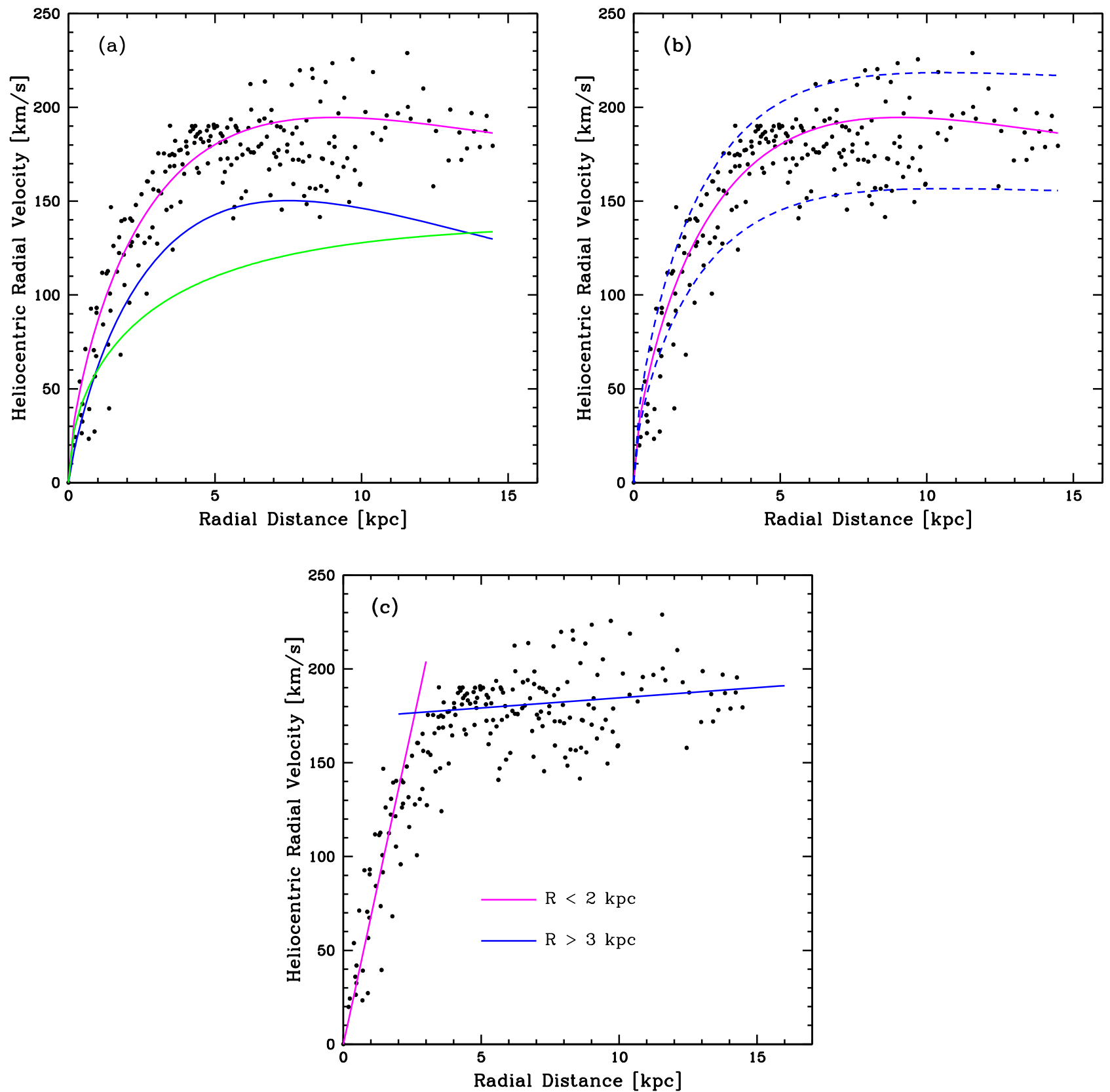

Figure 9. Heliocentric radial velocities corresponding to set "c." The chosen potentials correspond to the description of Barnes \& Hibbard (2009). In panel (a) the distribution is fitted considering a potential that includes an exponential disk (Freeman 1970) (blue line) and a halo component (Navarro et al. 1996) (green line). The resulting rotation curve is plotted in magenta. In panel (b) we plot the disk plus halo potential and two rotation curve envelopes (see text for more details). In panel (c) we include the linear fitting for two distinctive regions of the rotation curves: the central region of rigid rotation $(R<2 \mathrm{kpc})$ and the flat region $(R>3 \mathrm{kpc})$.

The $B$-band and $K$-band Tully-Fisher relations for these systems are flatter than the standard one derived for isolated galaxies. The same trend is observed in other interacting galaxy samples. More data on low-mass objects and 3D spectroscopic studies would be desirable for improving the T-F characterization of objects of this type.

From the kinematic data, we have derived a synthetic radial velocity distribution that serves as a reference for future numerical simulations. The synthetic rotation curve is near to solid body-like inside $4 \mathrm{kpc}$, and then is nearly flat within the radial range $5-15 \mathrm{kpc}$. The semi-amplitude of the curve is $\sim 190 \mathrm{~km} \mathrm{~s}^{-1} \mathrm{kpc}^{-1}$.

The distribution of the difference in position angle between the major axis of the main galaxies and the segment that connects the centers of main and satellite galaxies shows an excess of companions located toward the major axis of the main galaxy. A selection effect of M51 companions located nearer to the galaxy's major axis can be discarded because there is no correlation with the system's inclination. This indicates that in most of the systems in the M51 phase of the interaction, the companion's orbit has a large projection on the equatorial plane of the main galaxy. Consistently, the differences in radial velocity between main and satellite galaxies, normalized to the peak radial velocity of the velocity distribution of the main galaxy, indicates that the orbital motion of the satellite is within the range of amplitudes of the rotation curve of the main galaxy (see Figure 10). All the studied M51 systems are therefore 


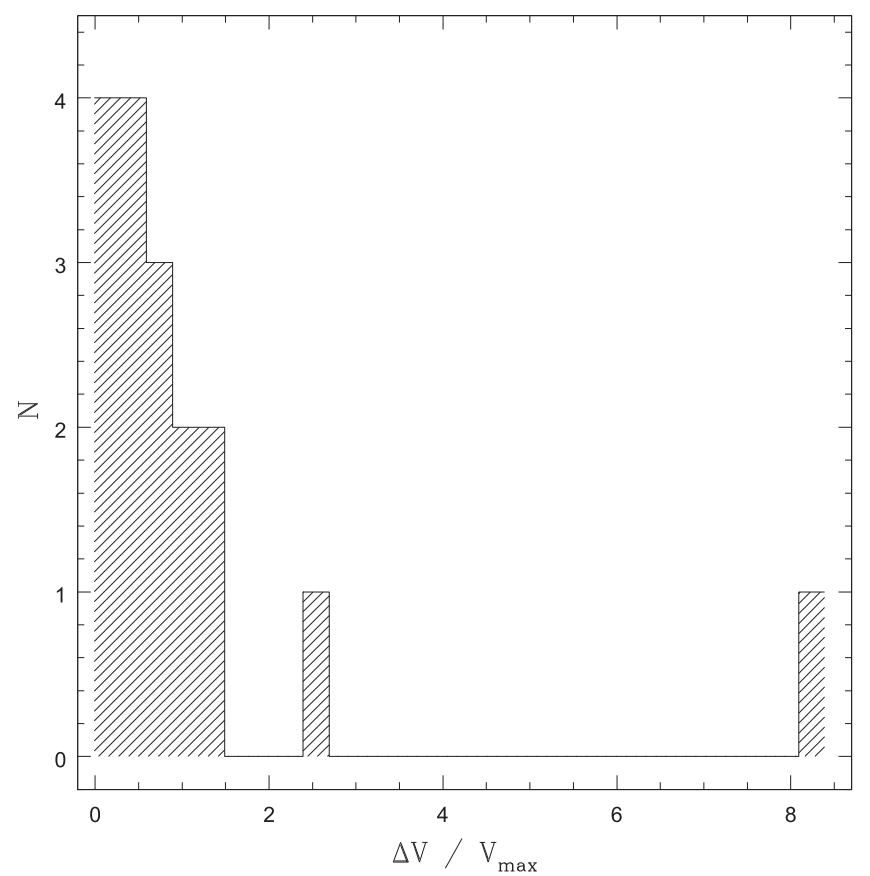

Figure 10. Distribution of differences in radial velocity between main and satellite galaxies, normalized to the amplitude of the main galaxy's radial velocity curve. Most of the companions have velocities consistent with orbital motion around the main galaxy. All the systems are gravitationally bound, except for the pair AM 2256-304, which seems to be a case of a high-velocity encounter.

gravitationally bound, except for one pair that seems to be a high-velocity encounter.

Despite the significant increase in data that resulted from the present work, there is a clear need for spectroscopic observations with higher spatial resolution and for a larger sample, in order to better constrain the kinematics and orbital parameters of M51-type galaxies.

It is a pleasure to thank the referee whose suggestions helped to improve the content and presentation of this paper. This work is based on observations obtained at Complejo Astronómico El Leoncito (San Juan). G.G. wants to specially thank Dr. Estela L. Agüero for her suggestion of studying the M51type systems and for her supervision at the beginning of this research. This research has made use of the NASA/IPAC Extragalactic Database (NED), which is operated by the Jet Propulsion Laboratory, California Institute of Technology, under contract with the National Aeronautics and Space Administration. This publication makes use of data products from the Two Micron All Sky Survey, which is a joint project of the University of Massachusetts and the Infrared Processing and Analysis Center/California Institute of Technology, funded by the National Aeronautics and Space Administration and the National Science Foundation. We acknowledge grant support from Secyt, UNC (05/N030) and grant support from CONICET (PIP 0523), ANPCyT (PICT 835). Supported by the Gemini Observatory, which is operated by the Association of Universities for Research in Astronomy, Inc., on behalf of the international Gemini partnership of the National Science Foundation (United States), the National Research Council (Canada), CONICYT (Chile), the Australian Research Council (Australia), Ministério da Ciência, Tecnologia e Inovação
(Brazil), and Ministerio de Ciencia, Tecnología e Innovación Productiva (Argentina).

\section{APPENDIX}

Here we list the functions employed in Section 3.7. The coefficients $a, b, c, d, e$, and $f$ are detailed in Table 7.

"Asymmetric Double Gaussian Cumulative" (ADC):

$$
\begin{aligned}
y= & \frac{a}{4}\left[1+\operatorname{erf}\left(\frac{x-b+c / 2}{d \sqrt{2}}\right)\right] \\
& \times\left[\frac{1}{2}-\frac{1}{2} \operatorname{erf}\left(\frac{x-b-c / 2}{\sqrt{2} e}\right)\right]+f .
\end{aligned}
$$

"Modified Gaussian" (GaussMod):

$$
y=e+a \exp \left[-\frac{1}{2}\left(\frac{|x-b|}{c}\right)^{d}\right] .
$$

"Symmetric Double Gaussian Cumulative" (SDC):

$$
y=\frac{a\left[1+\operatorname{erf}\left(\frac{x-b+c / 2}{d \sqrt{2}}\right)\right]\left[1-\operatorname{erf}\left(\frac{x-b-c / 2}{d \sqrt{2}}\right)\right]}{\left[1+\operatorname{erf}\left(\frac{c}{2 d \sqrt{2}}\right)\right]^{2}}+e
$$

where $\operatorname{erf}(x)$ is the Gauss error function,

$$
\operatorname{erf}(x)=\frac{2}{\sqrt{\pi}} \int_{0}^{x} e^{t^{2}} d t
$$

\section{REFERENCES}

Arp, H. 1966, ApJS, 14, 1

Arp, H., \& Madore, B. 1987, A Catalogue of Southern Peculiar Galaxies and Associations (Cambridge: Cambridge Univ. Press)

Barnes, J. E., \& Hibbard, J. E. 2009, AJ, 137, 3071

Barton, E. J., Geller, M. J., Bromley, B. C., van Zee, L., \& Kenyon, S. J. 2001, AJ, 121, 625

Bushouse, H. 1987, AJ, 320, 49

Calzetti, D., Kennicutt, R. C., Jr., Bianchi, L., et al. 2005, ApJ, 633, 871

Díaz, R. J., Rodrigues, I., Dottori, H., \& Carranza, G. 2001, AJ, 119, 111

Dopita, M. A., Pereira, M., Kewley, L. J., \& Capaccioli, M. 2002, ApJS, 143,47

Ellison, S. L., Patton, D. R., Simard, L., \& McConnachie, A. W. 2008, AJ, 135,1877

Freeman, K. C. 1970, ApJ, 160, 811

Fuentes-Carrera, I., Rosado, M., Amram, P., \& Laurikainen, E. 2007, A\&A, 466,847

Günthardt, G. I. 2009, PhD thesis, Famaf, Universidad Nacional de Córdoba

Hernquist, L. E. 1990, ApJ, 356, 359

Jokimäki, A., Orr, H., \& Russell, D. G. 2008, Ap\&SS, 315, 249

Karachentsev, I. D., Karachentseva, V. E., Huchtmeier, W. K., \& Makarov, D. I. 2004, AJ, 127, 2031

Karachentsev, I. D., \& Kutkin, A. M. 2005, AstL, 31, 5

Karachentseva, V. E., Mitronova, S. N., Mel'nik, O. V., \& Karachentsev, I. D. 2010, AstBu, 65, 1

Keel, W. C. 2004, ApJS, 106, 27

Klimanov, S., \& Reshetnikov, V. 2001, A\&A, 378, 428

Klimanov, S., Reshetnikov, V., \& Burenkov, A. 2002, AstL, 28, 579

Kudrya, Y. N., \& Karachentseva, V. E. 2012, Ap, 55, 435

Laurikainen, E., Salo, H., \& Aparicio, A. 1998, A\&AS, 129, 517

Maraston, C. 1998, MNRAS, 300, 872

Masters, K. L., Giovanelli, R., \& Haynes, M. P. 2003, AJ, 158, 174

Masters, K. L., Springob, C. M., \& Huchra, J. P. 2008, ApJ, 135, 1738

Navarro, J. F., Frenk, C. S., \& White, S. D. M. 1996, ApJ, 462, 563

Pierce, M. J., \& Tully, B. R. 1992, ApJ, 387, 47

Poggianti, B. M. 1997, A\&AS, 122, 399

Rampazzo, R., Plana, H., Amram, P., et al. 2005, MNRAS, 356, 1177 
Reshetnikov, V., \& Klimanov, S. 2003, AstL, 29, 429

Schlafly, E. F., \& Finkbeiner, D. P. 2011, ApJ, 737, 103

Smith, B. J., Struck, C., Hancock, M., et al. 2007, AJ, 133, 791

Thomasson, M. 2004, in ASP Conf. Ser. 320, The Neutral ISM in Starburst Galaxies, ed. S. Aalto, S. Hüttemeister, \& A. Pedlar, (San Francisco, CA: ASP), 81

Torres-Flores, S., Epinat, B., Amram, P., Plana, H., \& Mendes de Oliveira, C. 2011, MNRAS, 416, 1936
Torres-Flores, S., Mendes de Oliveira, C., Plana, H., B Amram, P., \& Epinat, B. 2013, MNRAS, 432, 3085

Tully, B. R., \& Pierce, M. J. 2000, ApJ, 533, 744

Tully, B. R., Pierce, M. J., Huang, J. S., et al. 1998, AJ, 115, 2264

van den Broek, A. C., van Driel, W., de Jong, T., et al. 1991, A\&AS, 91, 61

Vorontsov-Velyaminov, B. A. 1975, AZh, 52, 692

Woods, D. F., \& Geller, M. J. 2007, AJ, 134, 527

Zasov, A., \& Kopherskov, A. 2003, AstL, 29, 437 